\title{
VISCOUS, RESISTIVE MAGNETOROTATIONAL MODES
}

\author{
MARTIN E. PESSAH \\ School of Natural Sciences, Institute for Advanced Study, Princeton, NJ, 08540 \\ AND \\ CHI-KWAN CHAN \\ Institute for Theory and Computation, Harvard-Smithsonian Center for Astrophysics, 60 Garden Street, Cambridge, MA 02138 \\ Draft version May 30, 2018
}

\begin{abstract}
We carry out a comprehensive analysis of the behavior of the magnetorotational instability (MRI) in viscous, resistive plasmas. We find exact, non-linear solutions of the non-ideal magnetohydrodynamic (MHD) equations describing the local dynamics of an incompressible, differentially rotating background threaded by a vertical magnetic field when disturbances with wavenumbers perpendicular to the shear are considered. We provide a geometrical description of these viscous, resistive MRI modes and show how their physical structure is modified as a function of the Reynolds and magnetic Reynolds numbers. We demonstrate that when finite dissipative effects are considered, velocity and magnetic field disturbances are no longer orthogonal (as it is the case in the ideal MHD limit) unless the magnetic Prandtl number is unity. We generalize previous results found in the ideal limit and show that a series of key properties of the mean Reynolds and Maxwell stresses also hold for the viscous, resistive MRI. In particular, we show that the Reynolds stress is always positive and the Maxwell stress is always negative. Therefore, even in the presence of viscosity and resistivity, the total mean angular momentum transport is always directed outwards. We also find that, for any combination of the Reynolds and magnetic Reynolds numbers, magnetic disturbances dominate both the energetics and the transport of angular momentum and that the total mean energy density is an upper bound for the total mean stress responsible for angular momentum transport. The ratios between the Maxwell and Reynolds stresses and between magnetic and kinetic energy densities increase with decreasing Reynolds numbers for any magnetic Reynolds number; the lowest limit of both ratios is reached in the ideal MHD regime.
\end{abstract}

Subject headings: black hole physics — accretion, accretion disks — MHD — instability — turbulence

\section{INTRODUCTION}

The magnetorotational instability (MRI, Balbus \& Hawley 1991, 1998) has been widely studied in the inviscid and perfectly conducting, magnetohydrodynamic (MHD) limit. The departures from this idealized situation are usually parametrized according to the Reynolds $\operatorname{Re}=v l / \nu$ and magnetic Reynolds $\mathrm{Rm}=v l / \eta$ numbers, where $v$ and $l$ stand for the relevant characteristic velocity and lengthscale and $\nu$ and $\eta$ stand for the kinematic viscosity and resistivity. The ideal MHD regime is then formally identified with the limit $\mathrm{Re}, \mathrm{Rm} \rightarrow \infty$. There are many situations of interest in which the effects of dissipation need to be considered.

From the astrophysical point of view, accretion disks around young stellar objects constitute one of the most compelling reasons for investigating the MRI beyond the ideal limit. In particular, there is great interest in understanding to what extent can MHD turbulence driven by the MRI enable efficient angular momentum transport in cool, poorly conducting, protoplanetary disks (see, e.g. Blaes \& Balbus 1994; Jin 1996; Gammie 1996; Sano \& Miyama 1999; Salmeron \& Wardle 2005). Most of the studies addressing the effects of dissipation in non-ideal MRI have usually focused in inviscid, resistive plasmas. However, accretion disks are characterized by a wide range of magnetic Prandtl numbers, with $\mathrm{Pm}=\nu / \eta$ varying by several orders of magnitude

Electronic address: mpessah@ias.edu, ckchan@cfa.harvard.edu across the entire disk (see, e.g., Balbus \& Henri 2007). In order to understand the behavior of the MRI under these conditions it is necessary to relax the assumption of an inviscid plasma.

A large fraction of the shearing box simulations addressing the non-linear regime of the MRI have been carried out in the ideal MHD limit, i.e., without including explicit dissipation in the codes (see, e.g., Hawlev, Gammie. \& Balbus 1995; Brandenburg et al. 1995; Sano et al. 2004). However, even in the absence of explicit viscosity and resistivity, finite difference discretization leads to numerical diffusion/dissipation. Therefore, even in this type of simulations, it is necessary to understand the impact of these numerical artifacts that lead to departures from the ideal MHD regime and how similar they are when compared with physical (resolved) dissipation.

A handful of numerical studies with explicit resistivity but zero physical viscosity have been carried out in order to understand the effects of ohmic dissipation in the saturation of MRI-driven turbulence (see, e.g., Sano, Inutsuka, \& Miyama 1998; Sano \& Inutsuka 2001; Fleming, Stone, \& Hawley 2000; Sano et al. 2004; Turner, Sano, \& Dziourkevitch 2007). In particular, Sano \& Stone (2003) have shown that the saturation level of the stresses increases with increasing magnetic Reynolds number and seem to converge to an asymptotic value for magnetic Reynolds numbers larger than unity.

Recent work has pointed out problems with conver- 
gence in zero-net-flux numerical simulations of ideal MHD driven by the MRI (Pessah, Chan, \& Psaltis 2007; Fromang \& Papaloizou 2007) implying the necessity of incorporating explicit dissipation in the codes. Numerical studies with both resistivity and viscosity, in the presence of a mean vertical magnetic field (Lesur \& Longaretti 2007) and in the case of zero net flux (Fromang et al. 2007), have begun to uncover how the characteristics of fully developed MRI-driven turbulence depends on the Reynolds and magnetic Reynolds numbers. Even though the ranges in Reynolds and magnetic Reynolds numbers that can be currently addressed is still limited, the results obtained from the simulations suggest that the magnetic and kinetic energies contained in turbulent motions in the saturated regime depend on the values of the microphysical viscosity and resistivity. In particular, the mean angular momentum transport in the turbulent state increases with increasing magnetic Prandtl number.

From the experimental perspective, understanding the effects of non-vanishing resistivity and viscosity in the behavior of the MRI seems imperative, since the physical conditions achievable in the laboratory depart significantly from the ideal MHD regime (Ji, Goodman \& Kagevama 2001; Goodman \& Ji 2002; Sisan et al. 2004; Liu, Goodman, \& Ji 2006; Rüdiger, Schultz, Shalybkov 2003). Liquid metals (such as sodium, gallium, and mercury) are often characterized by rather low magnetic Prandtl numbers $\left(\mathrm{Pm} \simeq 10^{-5}\right.$ $\left.10^{-7}\right)$. Although the regime of Reynolds numbers involved is still orders of magnitude smaller than any astrophysical system with similar magnetic Prandtl numbers, MRI experiments offer one of the few prospects of studying anything close to MHD astrophysical processes in the laboratory.

A number of analyses addressing some aspects of the impact of viscosity and resistivity on the MRI in various dissipative limits appear scattered throughout the literature on theoretical, numerical, and experimental MRI. More recently, Lesaffre \& Balbus (2007) have found particular solutions of the viscous, resistive MHD equations (including even a cooling term) in the shearing box approximation. However, we are unaware of any comprehensive, systematic study addressing how the MRI behaves in viscous, resistive, differentially rotating magnetized plasmas for arbitrary combinations of the Reynolds and magnetic Reynolds numbers. The aim of this work is to carry out this analysis in detail.

The rest of paper is organized as follows. In $\S 2$ we state our assumptions. In $\S 3$, we solve the eigenvalue problem defined by the MRI for arbitrary Reynolds and magnetic Reynolds numbers. We provide closed analytical expressions for the eigenfrequencies and the associated eigenvectors. In $\S 4$, we address the unexplored physical structure of MRI modes for finite Reynolds and magnetic Reynolds numbers and derive simple analytical expressions that describe these modes in various asymptotic regimes. In $\S 6$ we calculate the correlations between magnetic and velocity MRI-driven perturbations that are related to angular momentum transport and energy densities. We find that some key results previously shown to hold in the ideal MHD limit (Pessah, Chan, \& Psaltis 2006) are also valid in the non-ideal regime. In particular, we show that even though the effectiveness with which the MRI disrupts the laminar flow depends on the Reynolds and magnetic Reynolds numbers, the instability always transports angular momentum outwards. We also find that magnetic perturbations dominate both the energetics and the transport of angular momentum for any combination of the Reynolds and magnetic Reynolds numbers. In $\S 7$ we summarize our findings and discuss the implications of our study.

\section{ASSUMPTIONS}

Let us consider a cylindrical, incompressible background characterized by an angular velocity profile $\Omega=\Omega(r) \check{z}$, threaded by a vertical magnetic field $\overline{\boldsymbol{B}}=\bar{B}_{z} \check{\boldsymbol{z}}$. We work in the shearing box approximation, which consist of a first order expansion in the variable $r-r_{0}$ of all the quantities characterizing the flow at the fiducial radius $r_{0}$. The goal of this expansion is to retain the most relevant terms governing the dynamics of the MHD fluid in a locally-Cartesian coordinate system co-orbiting and corrotating with the background flow with local (Eulerian) velocity $\boldsymbol{v}=r_{0} \Omega_{0} \check{\boldsymbol{\phi}}$. (For a more detailed discussion on this expansion see Goodman \& Xu 1994 and references therein.)

The equations governing the dynamics of an incompressible MHD fluid with constant kinematic viscosity $\nu$ and resistivity $\eta$ in the shearing box limit are given by

$$
\begin{aligned}
\frac{\partial \boldsymbol{v}}{\partial t}+(\boldsymbol{v} \cdot \boldsymbol{\nabla}) \boldsymbol{v} & =-2 \boldsymbol{\Omega}_{0} \times \boldsymbol{v}+q \Omega_{0}^{2} \boldsymbol{\nabla}\left(r-r_{0}\right)^{2} \\
& -\frac{1}{\rho} \boldsymbol{\nabla}\left(P+\frac{\boldsymbol{B}^{2}}{8 \pi}\right)+\frac{(\boldsymbol{B} \cdot \boldsymbol{\nabla}) \boldsymbol{B}}{4 \pi \rho}+\nu \boldsymbol{\nabla}^{2} \boldsymbol{v},
\end{aligned}
$$

$\frac{\partial \boldsymbol{B}}{\partial t}+(\boldsymbol{v} \cdot \boldsymbol{\nabla}) \boldsymbol{B}=(\boldsymbol{B} \cdot \boldsymbol{\nabla}) \boldsymbol{v}+\eta \boldsymbol{\nabla}^{2} \boldsymbol{B}$,

where $P$ is the pressure, $\rho$ is the (constant) density, the factor

$$
q \equiv-\left.\frac{d \ln \Omega}{d \ln r}\right|_{r_{0}},
$$

parametrizes the magnitude of the local shear, and we have defined the (locally-Cartesian) differential operator

$$
\boldsymbol{\nabla} \equiv \check{\boldsymbol{r}} \frac{\partial}{\partial r}+\frac{\check{\boldsymbol{\phi}}}{r_{0}} \frac{\partial}{\partial \phi}+\check{\boldsymbol{z}} \frac{\partial}{\partial z},
$$

where $\check{\boldsymbol{r}}, \check{\phi}$, and $\check{\boldsymbol{z}}$ are, coordinate-independent, orthonormal vectors corrotating with the background flow at $r_{0}$. The continuity equation reduces to $\boldsymbol{\nabla} \cdot \boldsymbol{v}=0$ and there is no need for an equation of state since the pressure can be determined from this condition.

We focus our attention on the dynamics of perturbations that depend only on the vertical coordinate. Under the current set of assumptions, these types of perturbations are known to exhibit the fastest growth rates in the ideal MHD case (Balbus \& Hawley 1992, 1998; Pessah \& Psaltis 2005). The equations governing the dynamics of these perturbations can be obtained by noting that the velocity and magnetic fields given by

$$
\begin{aligned}
\boldsymbol{v} & =\delta v_{r}(z) \check{\boldsymbol{r}}+\left[-q \Omega_{0}\left(r-r_{0}\right)+\delta v_{\phi}(z)\right] \check{\boldsymbol{\phi}}+\delta v_{z}(z) \check{\boldsymbol{z}},(5) \\
\boldsymbol{B} & =\delta B_{r}(z) \check{\boldsymbol{r}}+\delta B_{\phi}(z) \check{\boldsymbol{\phi}}+\left[\bar{B}_{z}+\delta B_{z}(z)\right] \check{\boldsymbol{z}},
\end{aligned}
$$

where the time dependence is implicit, constitute a family of exact, non-linear, solutions to the viscous, resistive MHD equations (1)-(2). As noted in Goodman \& Xu (1994), even in the dissipative case, the only non-linear terms, which are present through the perturbed magnetic energy density, are irrelevant in the case under consideration. 
We can further simplify equations (1) and (2) by removing the background shear flow ${ }^{1} \boldsymbol{v}_{\text {shear }}=-q \Omega_{0}\left(r-r_{0}\right) \check{\boldsymbol{\phi}}$ and by realizing that we can take $\delta v_{z}(z)=\delta B_{z}(z)=0$ without loss of generality. We then obtain

$$
\begin{aligned}
\frac{\partial}{\partial t} \delta v_{r} & =2 \Omega_{0} \delta v_{\phi}+\frac{\bar{B}_{z}}{4 \pi \rho} \frac{\partial}{\partial z} \delta B_{r}+\nu \frac{\partial^{2}}{\partial z^{2}} \delta v_{r} \\
\frac{\partial}{\partial t} \delta v_{\phi} & =-(2-q) \Omega_{0} \delta v_{r}+\frac{\bar{B}_{z}}{4 \pi \rho} \frac{\partial}{\partial z} \delta B_{\phi}+\nu \frac{\partial^{2}}{\partial z^{2}} \delta v_{\phi} \\
\frac{\partial}{\partial t} \delta B_{r} & =\bar{B}_{z} \frac{\partial}{\partial z} \delta v_{r}+\eta \frac{\partial^{2}}{\partial z^{2}} \delta B_{r} \\
\frac{\partial}{\partial t} \delta B_{\phi} & =-q \Omega_{0} \delta B_{r}+\bar{B}_{z} \frac{\partial}{\partial z} \delta v_{\phi}+\eta \frac{\partial^{2}}{\partial z^{2}} \delta B_{\phi}
\end{aligned}
$$

where the first term on the right hand side of equation (8) is related to the epicyclic frequency

$$
\kappa \equiv \sqrt{2(2-q)} \Omega_{0},
$$

at which the flow variables oscillate in a perturbed hydrodynamic disk. For Keplerian rotation the parameter is $q=3 / 2$ and thus the epicyclic frequency is $\kappa=\Omega_{0}$.

It is convenient to define the new variables $\delta b_{i} \equiv$ $\delta B_{i} / \sqrt{4 \pi \rho}$ for $i=r, \phi$, and introduce dimensionless quantities by considering the characteristic time- and length-scales set by $1 / \Omega_{0}$ and $\bar{B}_{z} /\left(\sqrt{4 \pi \rho} \Omega_{0}\right)$. The equations satisfied by the dimensionless perturbations, $\delta \tilde{v}_{i}, \delta \tilde{b}_{i}$, are then given by

$$
\begin{aligned}
& \partial_{\tilde{t}} \delta \tilde{v}_{r}=2 \delta \tilde{v}_{\phi}+\partial_{\tilde{z}} \delta \tilde{b}_{r}+\tilde{\nu} \partial_{\tilde{z}}^{2} \delta \tilde{v}_{r}, \\
& \partial_{\tilde{t}} \delta \tilde{v}_{\phi}=-(2-q) \delta \tilde{v}_{r}+\partial_{\tilde{z}} \delta \tilde{b}_{\phi}+\tilde{\nu} \partial_{\tilde{z}}^{2} \delta \tilde{v}_{\phi}, \\
& \partial_{\tilde{t}} \delta \tilde{b}_{r}=\partial_{\tilde{z}} \delta \tilde{v}_{r}+\tilde{\eta} \partial_{\tilde{z}}^{2} \delta \tilde{b}_{r}, \\
& \partial_{\tilde{t}} \delta \tilde{b}_{\phi}=-q \delta \tilde{b}_{r}+\partial_{\tilde{z}} \delta \tilde{v}_{\phi}+\tilde{\eta} \partial_{\tilde{z}}^{2} \delta \tilde{b}_{\phi},
\end{aligned}
$$

where $\tilde{t}$ and $\tilde{z}$ denote the dimensionless time and vertical coordinate, respectively.

The dynamics of ideal MRI modes, with $\nu=\eta=$ 0 , is completely determined by the dimensionless shear $q$ (Pessah, Chan, \& Psaltis 2006). The effects of viscous and resistive dissipation introduce two new dimensionless quantities that alter the characteristics and evolution of the MRI. With our choice of characteristic scales, it is natural to define the Reynolds and magnetic Reynolds numbers characterizing the MHD flow as ${ }^{2}$

$$
\begin{aligned}
\mathrm{Re} & \equiv \frac{v_{\mathrm{A} z}^{2}}{\nu \Omega_{0}}=\frac{1}{\tilde{\nu}}, \\
\mathrm{Rm} & \equiv \frac{v_{\mathrm{A} z}^{2}}{\eta \Omega_{0}}=\frac{1}{\tilde{\eta}},
\end{aligned}
$$

with associated magnetic Prandtl number

$$
\mathrm{Pm} \equiv \frac{\mathrm{Rm}}{\operatorname{Re}}=\frac{\tilde{\nu}}{\tilde{\eta}}=\frac{\nu}{\eta} .
$$

\footnotetext{
${ }^{1}$ In the shearing box approximation, the dependence of the background flow on the radial coordinate is strictly linear and therefore viscous dissipation does not affect its dynamics.

${ }^{2}$ In a compressible fluid, the sound speed, $c_{\mathrm{s}}$, provides another natural characteristic speed to define the Reynolds and magnetic Reynolds numbers. These definitions, e.g., $\mathrm{Re}^{\prime}$ and $\mathrm{Rm}^{\prime}$, are related to those provided in equations (16) and (17) via the plasma beta parameter $\beta=(2 / \Gamma)\left(c_{\mathrm{S}} / \bar{v}_{\mathrm{A} z}\right)^{2}$, with $\bar{v}_{\mathrm{A} z}=\bar{B}_{z} / \sqrt{4 \pi \rho}$ the Alfvén speed in the $z$ direction, simply by $\operatorname{Re}^{\prime}=(\Gamma \beta / 2) \operatorname{Re}$ and $\mathrm{Rm}^{\prime}=(\Gamma \beta / 2) \mathrm{Rm}$ for a polytropic equation of state $P=K \rho^{\Gamma}$, with $K$ and $\Gamma$ constants.
}

In order to simplify the notation, we drop hereafter the tilde denoting the dimensionless quantities. In the rest of the paper, all the variables are to be regarded as dimensionless, unless otherwise specified.

\section{THE EIGENVALUE PROBLEM FOR THE NON-IDEAL MRI : A FORMAL ANALYTICAL SOLUTION}

In this section we provide a complete analytical solution to the set of equations (12)-(15) as a function of the shear parameter $q$, (or, equivalently, the epicyclic frequency, $\kappa$ ) for any set of values $(\nu, \eta)$ defining the viscosity and resistivity.

It is convenient to work in Fourier space, as this provides the advantage of obtaining explicitly the basis of modes that is needed to construct the most general solution satisfying equations (12)- 15). Taking the Fourier transform of this set with respect to the $z$-coordinate, we obtain the matrix equation

$$
\partial_{t} \hat{\boldsymbol{\delta}}\left(k_{n}, t\right)=L \hat{\boldsymbol{\delta}}\left(k_{n}, t\right)
$$

where the vector $\hat{\boldsymbol{\delta}}\left(k_{n}, t\right)$ stands for

$$
\hat{\boldsymbol{\delta}}\left(k_{n}, t\right)=\left[\begin{array}{c}
\delta \hat{v}_{r}\left(k_{n}, t\right) \\
\delta \hat{v}_{\phi}\left(k_{n}, t\right) \\
\delta \hat{b}_{r}\left(k_{n}, t\right) \\
\delta \hat{b}_{\phi}\left(k_{n}, t\right)
\end{array}\right]
$$

and $L$ represents the matrix

$$
L=\left[\begin{array}{cccc}
-\nu k_{n}^{2} & 2 & i k_{n} & 0 \\
-(2-q) & -\nu k_{n}^{2} & 0 & i k_{n} \\
i k_{n} & 0 & -\eta k_{n}^{2} & 0 \\
0 & i k_{n} & -q & -\eta k_{n}^{2}
\end{array}\right]
$$

The functions denoted by $\hat{f}\left(k_{n}, t\right)$ correspond to the Fourier transform of the real functions, $f(z, t)$, and are defined via

$$
\hat{f}\left(k_{n}, t\right) \equiv \frac{1}{2 H} \int_{-H}^{H} f(z, t) e^{-i k_{n} z} d z,
$$

where we have assumed periodic boundary conditions at $z=$ $\pm H$, with $H$ being the (dimensionless) scale-height and $k_{n}$ the wavenumber in the $z$-coordinate,

$$
k_{n} \equiv \frac{n \pi}{H},
$$

where $n$ is an integer number. In order to simplify the notation, hereafter we denote these wavenumbers simply by $k$.

In order to solve the matrix equation (19), it is convenient to find the eigenvector basis, $\left\{\mathbf{e}_{j}\right\}$ with $j=1,2,3,4$, in which $L$ is diagonal. This basis exists for all values of the wavenumber $k$ (i.e., the rank of the matrix $L$ is equal to 4 , the dimension of the complex space) with the possible exception of a finite number of values of $k$. In this basis, the action of $L$ over the set $\left\{\mathbf{e}_{j}\right\}$ is equivalent to a scalar multiplication, i.e.,

$$
L_{\text {diag }} \mathbf{e}_{j}=\sigma_{j} \mathbf{e}_{j} \quad \text { for } \quad j=1,2,3,4,
$$

where $\left\{\sigma_{j}\right\}$ are complex scalars.

\subsection{Eigenvalues}

In the eigenvector basis, the matrix $L$ has a diagonal representation $L_{\text {diag }}=\operatorname{diag}\left(\sigma_{1}, \sigma_{2}, \sigma_{3}, \sigma_{4}\right)$. The eigenvalues $\left\{\sigma_{j}\right\}$, 

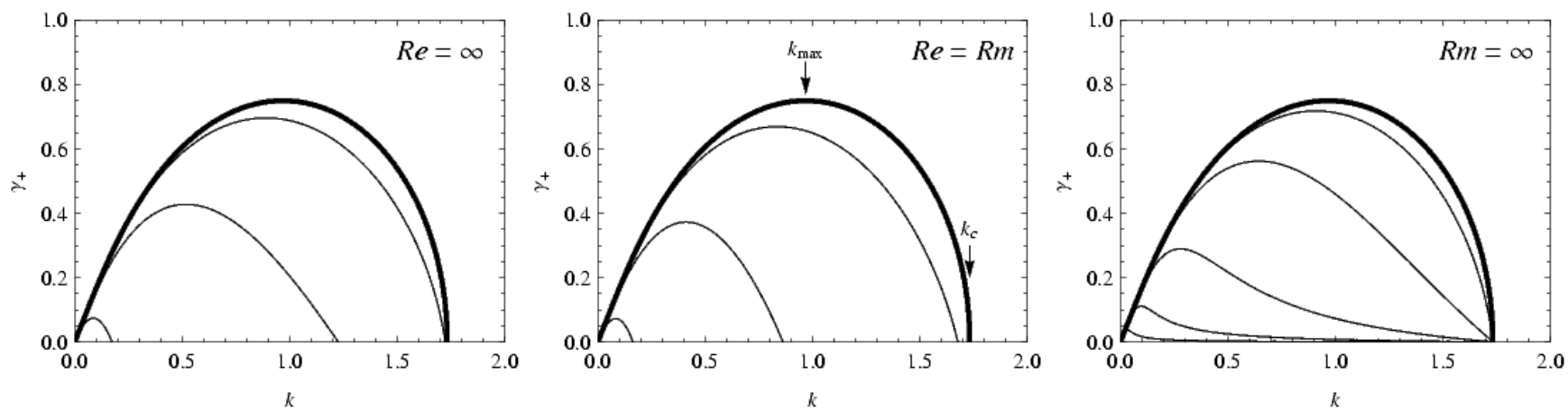

FIG. 1.- Growth rates $\gamma_{+}$, eq. (44), as a function of the vertical wavenumber $k$ for different combinations of Reynolds and magnetic Reynolds numbers for Keplerian rotation. In all three panels, the thick solid line corresponds to the ideal MHD limit, i.e., Re, $\mathrm{Rm} \rightarrow \infty$. For any combination of the Reynolds and magnetic Reynolds numbers, the growth rate has a well defined, single maximum $\gamma_{\max }$ that corresponds to the most unstable mode $k_{\max }$. The range of unstable modes, $0<k<k_{\mathrm{c}}$, is always finite, the critical wavenumber $k_{\mathrm{c}}$ satisfies eq. 25] when $\sigma \equiv 0$, see $\S 4.1$ Left: Growth rate $\gamma_{+}$for different values of the magnetic Reynolds number in the inviscid limit, i.e., Re $\rightarrow \infty$. The thin solid lines, in decreasing order, correspond to Rm $=10,1,0.1$. Middle: Growth rate $\gamma_{+}$for magnetic Prandtl number $\mathrm{Pm}=\mathrm{Rm} / \mathrm{Re}=1$. The thin solid lines, in decreasing order, correspond to $\mathrm{Re}=\mathrm{Rm}=10,1,0.1$. In all of the cases shown in the left and middle panels, the critical wavenumber, $k_{\mathrm{c}}$, below which unstable modes can exist decreases with increasing resistivity, see $\S 4.3$ and $\S 4.4$ for analytic expressions of these marginally stable modes. Right: Growth rate $\gamma_{+}$for different values of the Reynolds number in the ideal conductor limit, i.e., $\mathrm{Rm} \rightarrow \infty$. The various curves, in decreasing order, correspond to $\mathrm{Re}=10,1, \ldots, 10^{-3}$. In this case, the range of unstable modes is insensitive to the Reynolds number, all the modes with wavenumbers shorter than $k_{\mathrm{c}}=\sqrt{2 q}$ are unstable, see $\delta 4.2$ It is evident that the growth rates and the characteristic scales, both $k_{\max }$ and $k_{\mathrm{c}}$, are more sensitive to changes in the resistivity than to changes in the viscosity. The simultaneous analysis of all three panels leads to the conclusion that viscous, resistive modes with magnetic Prandtl number equal to unity resemble more closely inviscid, resistive modes rather than viscous, conductive ones, see $\S 4.4$ for the explanation of this behavior.

with $j=1,2,3,4$, are the roots of the characteristic polynomial associated with $L$, i.e., the dispersion relation associated with the non-ideal MRI, which can be written in compact form as

$$
\left(k^{2}+\sigma_{\nu} \sigma_{\eta}\right)^{2}+\kappa^{2}\left(k^{2}+\sigma_{\eta}^{2}\right)-4 k^{2}=0,
$$

where we have defined the quantities

$$
\begin{gathered}
\sigma_{\nu} \equiv \sigma+\nu k^{2}, \\
\sigma_{\eta} \equiv \sigma+\eta k^{2} .
\end{gathered}
$$

The dispersion relation (25) is a fourth order polynomial with non-zero coefficients in $\sigma$ and $\sigma^{3}$. In order to find its roots it is convenient to take this polynomial to its depressed form. This can be achieved by defining the new variables $\sigma_{\mu}$ and $\mu$ such that ${ }^{3}$

$$
\begin{aligned}
\sigma_{\mu} & \equiv \frac{1}{2}\left(\sigma_{\nu}+\sigma_{\eta}\right), \\
\mu & \equiv \frac{1}{2}(\nu-\eta) k^{2} .
\end{aligned}
$$

The resulting polynomial can then be written as

$$
\sigma_{\mu}^{4}+\alpha \sigma_{\mu}^{2}+\beta \sigma_{\mu}+\lambda=0
$$

where the coefficients $\alpha, \beta$, and $\lambda$ are given by

$$
\begin{aligned}
\alpha & \equiv 2\left(k^{2}-\mu^{2}\right)+\kappa^{2}, \\
\beta & \equiv-2 \mu \kappa^{2}, \\
\lambda & \equiv\left(k^{2}-\mu^{2}\right)^{2}+\kappa^{2}\left(k^{2}+\mu^{2}\right)-4 k^{2} .
\end{aligned}
$$

The solutions to equation (30) are

$$
\sigma_{\mu}= \pm_{a}\left(-\Lambda \mp_{b} \sqrt{\Delta}\right)^{1 / 2} \pm_{b} \frac{\beta}{4 \sqrt{\Delta}}
$$

where the subscripts $a$ and $b$ in the "+" and "-" signs label the four possible combinations of signs and we have defined

\footnotetext{
${ }^{3}$ A physical interpretation of the variable $\mu$ is provided in $\S 3.3$
}

the quantities ${ }^{4}$

$$
\begin{aligned}
& \Lambda=\frac{3 \alpha}{4}+\frac{y}{2}, \\
& \Delta=(y+\alpha)^{2}-\lambda,
\end{aligned}
$$

and $y$ is any of the solutions to the cubic equation

$$
y^{3}+\frac{5 \alpha}{2} y^{2}+\left(2 \alpha^{2}-\lambda\right) y+\left(\frac{\alpha^{3}}{2}-\frac{\alpha \lambda}{2}-\frac{\beta^{2}}{8}\right)=0,
$$

which has closed analytic solutions

$$
y=-\frac{5}{6} \alpha+\frac{1}{3} \frac{P}{U}-U
$$

with

$$
\begin{aligned}
& P=-\frac{\alpha^{2}}{12}-\lambda, \\
& Q=-\frac{\alpha^{3}}{108}+\frac{\alpha \lambda}{3}-\frac{\beta^{2}}{8}, \\
& U=\left(\frac{Q}{2} \pm \sqrt{\frac{Q^{2}}{4}+\frac{P^{3}}{27}}\right)^{1 / 3} .
\end{aligned}
$$

Note that the choice of either sign in $U$ is immaterial.

It is now trivial to write the solutions of the dispersion relation (25), $\sigma$, in terms of the variable $\sigma_{\mu}$. Using equations (26) - 28) we obtain

$$
\sigma=\sigma_{\mu}-\frac{1}{2}(\nu+\eta) k^{2}
$$

The eigenfrequencies of the viscous, resistive MRI modes are then given by

$$
\begin{aligned}
\sigma & = \pm_{a}\left(-\Lambda \mp_{b} \sqrt{\Delta}\right)^{1 / 2} \\
& -\frac{\nu}{2} k^{2}\left(1 \pm_{b} \frac{\kappa^{2}}{2 \sqrt{\Delta}}\right)-\frac{\eta}{2} k^{2}\left(1 \mp_{b} \frac{\kappa^{2}}{2 \sqrt{\Delta}}\right) .
\end{aligned}
$$

${ }^{4}$ Defining the quantities $\Lambda$ and $\Delta$ in this way allows us to show explicitly that in the limit $\nu, \eta \rightarrow 0$ the solutions to equation 25 converge smoothly to the solutions found in the ideal MHD case (see Appendix A). 

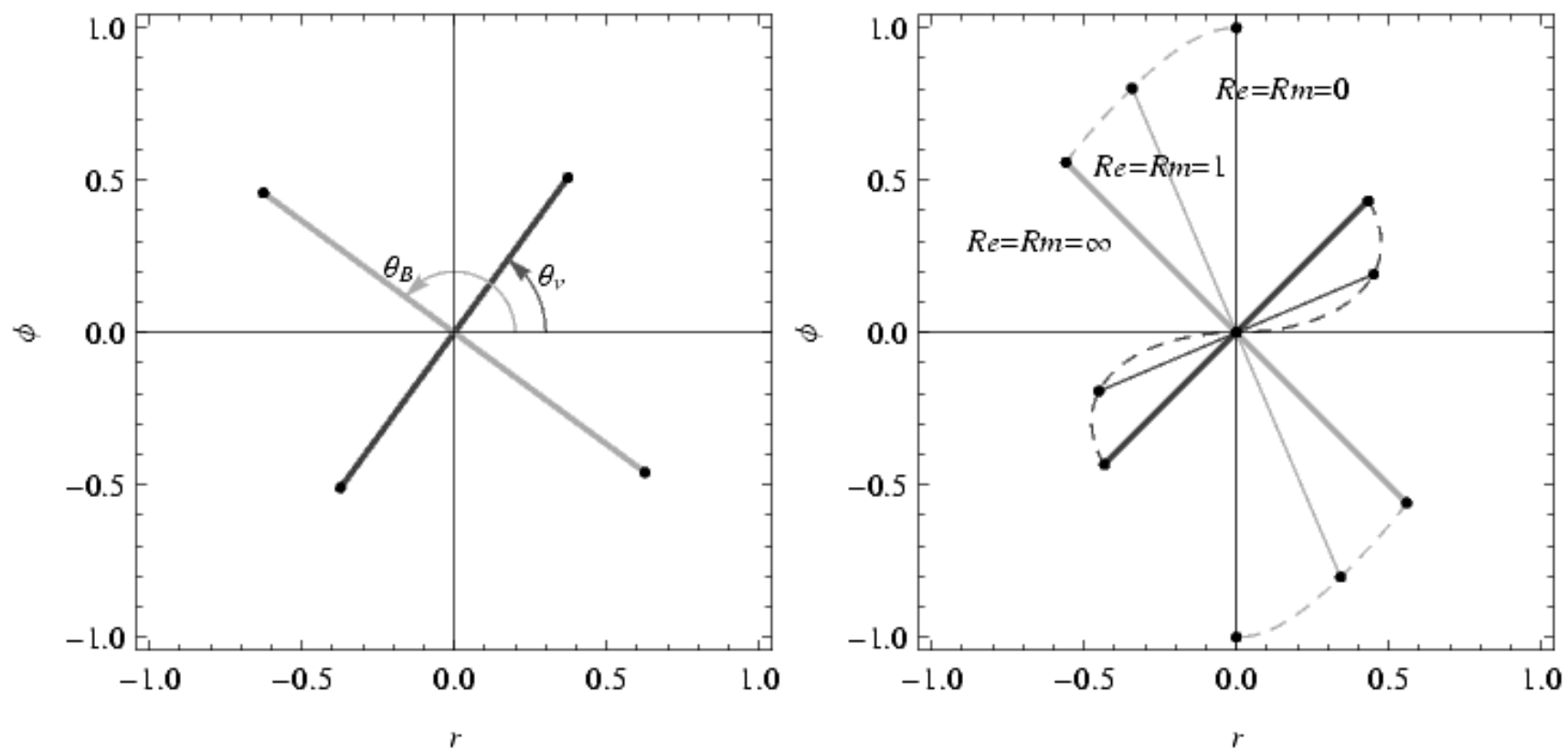

FIG. 2.- Left: Geometrical representation of the velocity field (black) and magnetic field (gray) perturbations for viscous, resistive MRI modes. Note that this is a projection of a single mode, which is inherently three-dimensional, onto the disk mid-plane $(r, \phi, z=0)$. The velocity and magnetic field components are always out of phase in the vertical direction $z$ by $\pi / 4$, see eq. (63). The angles $\theta_{\mathrm{v}}$ and $\theta_{\mathrm{b}}$, defined in eqs. (50) and (51), respectively, correspond to the physical angles defining the planes (perpendicular to the disk midplane) containing the MRI-driven perturbations, see eqs. 64) and (65). The relative magnitude of velocity and magnetic field perturbations is determined by eq. (54). Right: Evolution of the geometrical representation of the fastest-growing, non-ideal MRI mode, with associated wavenumber $k_{\max }$, with magnetic Prandtl number equal to unity, as a function of the Reynolds/magnetic Reynolds number. When the Reynolds/magnetic Reynolds number varies according to $\mathrm{Re}=\mathrm{Rm}: \infty \rightarrow 0$, the angles evolve according to $\theta_{\mathrm{v}}: \pi / 4 \rightarrow 0$ and $\theta_{\mathrm{b}}: 3 \pi / 4 \rightarrow \pi / 2$ and the relative amplitude of the perturbations evolves according to $b_{0} / v_{0}: 5 / 3 \rightarrow \infty$. Note that the velocity and magnetic field perturbations are always orthogonal for $\mathrm{Pm}=1$, see eq. 68).

\subsection{Four classes of solutions}

All of the quantities $\Lambda, \Delta$, and $y$, depend on the viscosity $\nu$ and the resistivity $\eta$ only through $\mu^{2} \propto(\nu-\eta)^{2}$. This has a series of important implications, in particular, there is always a range of wavenumbers for which the discriminant in equation (43) is positive, i.e., $\sqrt{\Delta}-\Lambda>0$. It can also be seen that the last two terms between parentheses in equation (43) are always positive, i.e., $\sqrt{\Delta} \geq \kappa^{2} / 2$, and thus they always produce damping. Because of this, we can classify the modes in four types: two (damped) growing and decaying "unstable" modes with eigenvalues

$$
\begin{aligned}
\gamma_{ \pm} & = \pm(\sqrt{\Delta}-\Lambda)^{1 / 2} \\
& -\frac{\nu}{2} k^{2}\left(1-\frac{\kappa^{2}}{2 \sqrt{\Delta}}\right)-\frac{\eta}{2} k^{2}\left(1+\frac{\kappa^{2}}{2 \sqrt{\Delta}}\right)
\end{aligned}
$$

and two (damped) "oscillatory" modes with eigenvalues

$$
\begin{aligned}
i \omega_{ \pm} & = \pm i(\sqrt{\Delta}+\Lambda)^{1 / 2} \\
& -\frac{\nu}{2} k^{2}\left(1+\frac{\kappa^{2}}{2 \sqrt{\Delta}}\right)-\frac{\eta}{2} k^{2}\left(1-\frac{\kappa^{2}}{2 \sqrt{\Delta}}\right) .
\end{aligned}
$$

We arbitrarily label these eigenvalues as

$$
\sigma_{1} \equiv \gamma_{+}, \quad \sigma_{2} \equiv \gamma_{-}, \quad \sigma_{3} \equiv i \omega_{+}, \quad \sigma_{4} \equiv i \omega_{-} .
$$

Figure 1 shows the growth rate $\gamma_{+}$as a function of the vertical wavenumber $k$ for different combinations of the Reynolds and magnetic Reynolds numbers for Keplerian rotation. These growth rates are more sensitive to changes in the resistivity than to changes in the viscosity. A qualitative understanding of this behavior can be obtained by realizing that viscosity tends to quench the instability, without altering the large scale magnetic field. Thus, as long as the resistivity is negligible, the range of unstable lenghtscales are the same in both ideal and viscous, perfectly conducting fluids. On the other hand, resistivity tends to destroy the magnetic field at small scales having a stronger impact on the stability of the perturbations at these scales.

Mathematically, the asymmetric response of the growth rate to changes in the viscosity $\nu$ or the resistivity $\eta$ originates in the different functional form of the terms that contribute to produce damping, i.e., $\left(1-\kappa^{2} / 2 \sqrt{\Delta}\right)$ and $\left(1+\kappa^{2} / 2 \sqrt{\Delta}\right)$, in the exponential growth characterized by $\gamma_{+}$in equation (44). If the oscillatory modes, $\omega_{ \pm}$in equation (45), are considered instead, the roles of the plus and minus signs in these terms are interchanged. From this analysis we can infer that the "oscillatory" mode is affected (damped) more strongly by viscosity than by resistivity.

The simultaneous analysis of the various panels in Figure 1 leads to the conclusion that viscous, resistive unstable modes with magnetic Prandtl number equal to unity resemble more closely inviscid, resistive modes rather than viscous, conductive ones. In $\S 4$ we provide analytical expressions to support this conclusion.

\subsection{Normalized Eigenvectors: Geometrical Representation}

The set of normalized eigenvectors, $\left\{\mathbf{e}_{\sigma_{j}}\right\}$, associated with the eigenvalues (46) are given by

$$
\mathbf{e}_{\sigma_{j}} \equiv \frac{\mathbf{e}_{j}}{\left\|\mathbf{e}_{j}\right\|} \quad \text { for } \quad j=1,2,3,4
$$



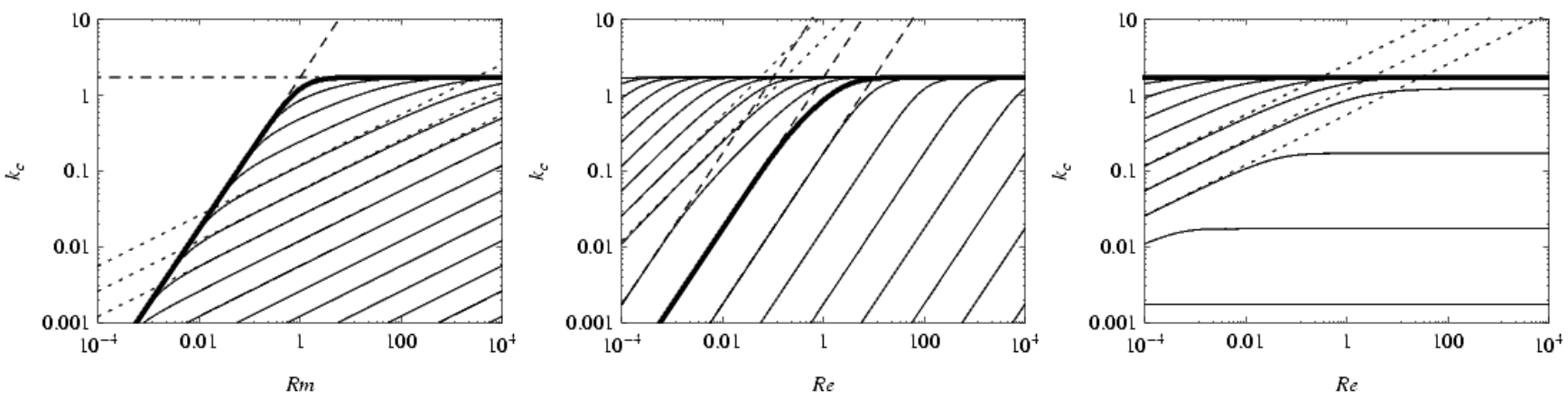

FIG. 3.- Critical wavenumber $k_{\mathrm{c}}$, see eq. 70, corresponding to the marginally stable MRI mode for Keplerian rotation in different dissipative regimes. The horizontal lines at $k_{\mathrm{c}}=\sqrt{3}$ represent the ideal MHD limit, eq. 76. Left: Critical wavenumber $k_{\mathrm{c}}$ as a function of the magnetic Reynolds number for different values of the Reynolds number. The thick solid line denotes the inviscid limit, i.e., $\operatorname{Re} \rightarrow \infty$. Note that eq. [87] describes this curve exactly. The thin solid lines, in decreasing order, correspond to Re $=1,0.1, \ldots$. For small magnetic Reynolds number $k_{\mathrm{c}} \propto \mathrm{Rm}$, see eq. 87]. For finite Reynolds numbers, such that $\operatorname{Re} \mathrm{Rm} \lesssim 1$ there is a transition between the regimes $\mathrm{Rm} \ll 1$ and $\mathrm{Rm} \gg 1$ such that $k_{\mathrm{c}} \propto \mathrm{Rm}^{1 / 3}$, see eq. (98). Middle: Critical wavenumber $k_{\mathrm{C}}$ as a function of the Reynolds number for different magnetic Prandtl numbers. Pm increases/decreases by an order of magnitude for each curve to the left/right of the thick solid line denoting the $\mathrm{Pm}=1$ case. The dashed lines $k_{\mathrm{c}} \propto \mathrm{RePm}=\mathrm{Rm}$ are calculated according to eq. 877, which gives the correct result even for $\mathrm{Pm}>1$, provided that the Reynolds number is sufficiently small. The dotted lines $k_{\mathrm{c}} \propto(\mathrm{Re} \mathrm{Rm})^{1 / 3}$ are calculated according to eq. 98$)$. Right: Critical wavenumber $k_{\mathrm{c}}$ as a function of the Reynolds number for different values of the magnetic Reynolds number. The thick solid line corresponds to the ideal conductor limit, i.e., $\mathrm{Rm} \rightarrow \infty$. The thin solid lines, in decreasing order, correspond to Re $=10^{5}, 10^{4}, \ldots, 10^{-3}$. For small Reynolds numbers $k_{\mathrm{c}} \propto \mathrm{Re}^{1 / 3}$, see eq. 98, while for Reynolds numbers larger than a few, the critical wavenumber is independent of Re regardless of the value of Rm.

where

$$
\mathbf{e}_{j}(k)=\left[\begin{array}{c}
\sigma_{\eta j} \\
\left(k^{2}+\sigma_{\nu j} \sigma_{\eta j}\right) / 2 \\
i k \\
-i k\left[2 \sigma_{\eta j}+q(\nu-\eta) k^{2}\right] /\left(k^{2}+\sigma_{\nu j} \sigma_{\eta j}\right)
\end{array}\right]
$$

$\sigma_{\nu j}=\sigma_{j}+\nu k^{2}, \sigma_{\eta j}=\sigma_{j}+\eta k^{2}$, and the norms are given by

$$
\left\|\mathbf{e}_{j}\right\| \equiv\left[\sum_{l=1}^{4} \mathrm{e}_{j}^{l} \mathrm{e}_{j}^{l *}\right]^{1 / 2}
$$

Here, $\mathrm{e}_{j}^{l}$ is the $l$-th component of the (unnormalized) eigenvector associated with the eigenvalue $\sigma_{j}$.

The set of four eigenvectors $\left\{\mathbf{e}_{\sigma_{j}}\right\}$, together with the set of complex scalars $\left\{\sigma_{j}\right\}$ in equation (46), constitute the full solution to the eigenvalue problem defined by the MRI for any combination of the Reynolds and magnetic Reynolds numbers.

A geometrical representation of the eigenvectors 47 can be brought to light by defining the angles $\theta_{\mathrm{v} j}$ and $\theta_{\mathrm{b} j}$ according to

$$
\begin{aligned}
& \tan \theta_{\mathrm{v} j} \equiv \frac{e_{j}^{2}}{e_{j}^{1}}=\frac{k^{2}+\sigma_{\nu j} \sigma_{\eta j}}{2 \sigma_{\eta j}}, \\
& \tan \theta_{\mathrm{b} j} \equiv \frac{e_{j}^{4}}{e_{j}^{3}}=-\frac{2 \sigma_{\eta j}+q(\nu-\eta) k^{2}}{k^{2}+\sigma_{\nu j} \sigma_{\eta j}} .
\end{aligned}
$$

It is important to remark that each of the four eigenvectors define, in principle, four sets of angles $\left\{\theta_{\mathrm{v} j}, \theta_{\mathrm{b} j}\right\}$, for $j=$ $1,2,3,4$. We label the angles associated with the different types of modes discussed in $\S 3.2$ according to

$$
\theta_{\mathrm{v} 1} \equiv \theta_{\mathrm{v}}^{\gamma_{+}}, \quad \theta_{\mathrm{v} 2} \equiv \theta_{\mathrm{v}}^{\gamma_{-}}, \quad \theta_{\mathrm{v} 3} \equiv \theta_{\mathrm{v}}^{\omega_{+}}, \quad \theta_{\mathrm{v} 4} \equiv \theta_{\mathrm{v}}^{\omega_{-}},
$$

with similar definitions corresponding to $\theta_{\mathrm{b} j}$ for $j=1,2,3,4$. Note that these angles are defined in spectral space and depend, in general, on the wavenumber $k$, the epicyclic frequency, $\kappa$, the viscosity $\nu$, and the resistivity $\eta$. The angles associated with the modes labeled by $\gamma_{+}$and $\gamma_{-}$are always real while the ones associated with the modes $\omega_{+}$and $\omega_{-}$are in general complex. For the sake of brevity, in what follows we will refer to the set of angles describing unstable MRI modes $\left\{\theta_{\mathrm{v}}^{\gamma_{+}}, \theta_{\mathrm{b}}^{\gamma_{+}}\right\}$simply as $\left\{\theta_{\mathrm{v}}, \theta_{\mathrm{b}}\right\}$.

A normalized version of the MRI eigenvectors can be obtained by multiplying the set of vectors in equation (48) by the amplitudes

$$
A_{j} \equiv \sqrt{\frac{2}{q\left(k^{2}+\sigma_{\eta j}^{2}\right)}} \frac{v_{0}}{\sqrt{v_{0}^{2}+b_{0}^{2}}} .
$$

where we have defined

$b_{0} \equiv \frac{2 k v_{0}}{k^{2}+\sigma_{\nu j} \sigma_{\eta j}}\left(1+\frac{(\nu-\eta)\left[4 \sigma_{\eta j}+q(\nu-\eta) k^{2}\right] k^{2}}{2\left(k^{2}+\sigma_{\eta j}^{2}\right)}\right)^{1 / 2}$

where, for the sake of simplicity, we have omitted the subscript $j$ on the left hand side. The expressions for the normalized eigenvectors $\left\{\mathbf{e}_{\sigma_{j}}\right\}$, for $j=1,2,3,4$, are then given by

$$
\mathbf{e}_{\sigma_{j}}(k)=\frac{1}{\sqrt{v_{0}^{2}+b_{0}^{2}}}\left[\begin{array}{c}
v_{0} \cos \theta_{\mathrm{v} j} \\
v_{0} \sin \theta_{\mathrm{v} j} \\
i b_{0} \cos \theta_{\mathrm{b} j} \\
i b_{0} \sin \theta_{\mathrm{b} j}
\end{array}\right]
$$

It is interesting to note that in this geometric representation the dispersion relation (25) can be obtained from the trigonometric identity

$$
\cos ^{2} \theta_{\mathrm{v} j}+\sin ^{2} \theta_{\mathrm{v} j}=1
$$

where the expressions for

$$
\begin{aligned}
\cos \theta_{\mathrm{v} j} & =\sqrt{\frac{2 \sigma_{\eta j}^{2}}{q\left(k^{2}+\sigma_{\eta j}^{2}\right)}}, \\
\sin \theta_{\mathrm{v} j} & =\frac{k^{2}+\sigma_{\nu j} \sigma_{\eta j}}{\sqrt{2 q\left(k^{2}+\sigma_{\eta j}^{2}\right)}}
\end{aligned}
$$



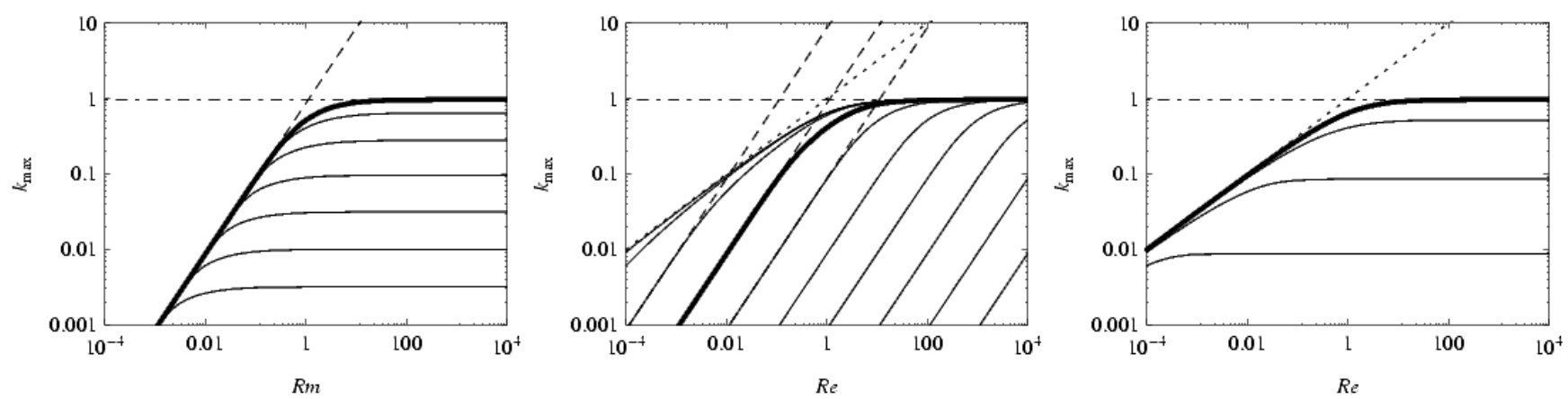

FIG. 4.- Wavenumber $k_{\max }$ corresponding to the fastest growing non-ideal MRI modes for Keplerian rotation in different dissipative regimes. The dot-dashed horizontal lines at $k_{\max }=\sqrt{15 / 16}$ represent the ideal MHD limit, eq. 85. Left: Fastest growing mode $k_{\max }$ as a function of the magnetic Reynolds number for different values of the Reynolds number. The thick solid line denotes the inviscid limit, i.e., $\operatorname{Re} \rightarrow \infty$. The thin solid lines, in decreasing order, correspond to $\mathrm{Re}=1,0.1, \ldots, 10^{-5}$. For magnetic Reynolds numbers larger than unity, this wavenumber is independent of Rm regardless of the value of Re. The dashed line, calculated according to eq. 90, provides the correct asymptotic limit $k_{\max } \propto \mathrm{Rm}$ for small magnetic Reynolds numbers. Middle: Fastest growing mode $k_{\max }$ as a function of the Reynolds number for different values of the magnetic Prandtl number. From left to right, the curves correspond to $\mathrm{Pm}=10^{3}, 10^{2}, \ldots, 1$ (thick solid line), $\ldots, 10^{-6}$. The dashed lines $k_{\max } \propto \mathrm{Re} \mathrm{Pm}=\mathrm{Rm}$ are calculated according to eq. (90), which leads to the correct result even for Pm $\gtrsim 1$ provided that the Reynolds number is sufficiently small. The dotted line $k_{\max } \propto \operatorname{Re}^{1 / 2}$ results from eq. [101. Right: Fastest growing mode $k_{\max }$ as a function of the Reynolds number for different values of the magnetic Reynolds number. The thick solid line corresponds to the ideal conductor limit, i.e., $\mathrm{Rm} \rightarrow \infty$. The thin solid lines, in decreasing order, correspond to $\mathrm{Rm}=1,0.1,0.01$. For Reynolds numbers larger than unity, the growth rate is independent of Re regardless of the value of Rm. The dotted line, calculated according to eq. 101, provides the correct asymptotic limit $k_{\max } \propto \mathrm{Re}^{1 / 2}$ for small Reynolds number.

can be obtained from the definition of the angle $\theta_{\mathrm{v} j}$ in equation (50).

\subsection{Temporal Evolution}

In physical space, the most general solution to the set of equations (12) $-(15)$, i.e.,

$$
\boldsymbol{\delta}(z, t)=\left[\begin{array}{l}
\delta v_{r}(z, t) \\
\delta v_{\phi}(z, t) \\
\delta b_{r}(z, t) \\
\delta b_{\phi}(z, t)
\end{array}\right]
$$

evolves in time according to

$$
\boldsymbol{\delta}(z, t) \equiv \sum_{k} \hat{\boldsymbol{\delta}}(k, t) e^{i k z}
$$

where

$$
\hat{\boldsymbol{\delta}}(k, t)=\sum_{j=1}^{4} a_{j}(k, 0) e^{\sigma_{j} t} \mathbf{e}_{\sigma_{j}},
$$

with $\left\{\sigma_{j}\right\}$ and $\left\{\mathbf{e}_{\sigma_{j}}\right\}$, for $j=1,2,3,4$, given by equations (46) and (55). The initial conditions $\boldsymbol{a}(k, 0)$ are related to the initial spectrum of perturbations, $\hat{\boldsymbol{\delta}}(k, 0)$, via $\boldsymbol{a}(k, 0)=$ $Q^{-1} \hat{\boldsymbol{\delta}}(k, 0)$. Here, $Q^{-1}$ is the matrix for the change of coordinates from the standard basis to the normalized eigenvector basis $^{5}$ and can be obtained by calculating the inverse of the matrix

$$
Q=\left[\begin{array}{llll}
\mathbf{e}_{\sigma_{1}} & \mathbf{e}_{\sigma_{2}} & \mathbf{e}_{\sigma_{3}} & \mathbf{e}_{\sigma_{4}}
\end{array}\right]
$$

The temporal evolution of a single MRI-unstable mode in physical space can be obtained from a linear combination of $\mathbf{e}_{\sigma_{j}}(k)$ and $\mathbf{e}_{\sigma_{j}}(-k)$ as defined in equation (55). In particular,

\footnotetext{
${ }^{5}$ The eigenvectors 55, are not in general orthogonal, i.e., $\mathbf{e}_{\sigma_{j}} \cdot \mathbf{e}_{\sigma_{j^{\prime}}} \neq 0$ for $j \neq j^{\prime}$. If desired, an orthogonal basis can be constructed using the GramSchmidt orthogonalization procedure (see, e.g., Hoffman \& Kunze 1971).
}

setting $a_{1}(k, 0)=a_{1}^{*}(-k, 0)=-i / \sqrt{2}$ in equation (60) and substituting the result in equation (61) we obtain

$$
\boldsymbol{\delta}(z, t)=\frac{\sqrt{2} e^{\gamma+t}}{\sqrt{v_{0}^{2}+b_{0}^{2}}}\left[\begin{array}{c}
v_{0} \cos \theta_{\mathrm{v}} \sin (k z) \\
v_{0} \sin \theta_{\mathrm{v}} \sin (k z) \\
b_{0} \cos \theta_{\mathrm{b}} \cos (k z) \\
b_{0} \sin \theta_{\mathrm{b}} \cos (k z)
\end{array}\right] .
$$

These solutions are of particular importance for the linear late-time evolution of MRI modes. Note that any reasonable spectrum of initial perturbations of the type used in numerical simulations of shearing boxes will have a non-zero component along the unstable eigenvector $e_{\sigma_{1}}$. If the value of the magnetic field is such that the MRI can be excited for given values of the viscosity and resistivity then the exponentially growing perturbations in physical space will evolve towards a mode of the form (63) dominated by the lengthscale $k=k_{\max }$ for which the growth rate reaches its maximum value $\gamma_{\max }$.

Note that if a perturbation in physical space is composed by a single mode of the type described in $\S 3.2$ no matter which class, then the angles defined in equations (50) and (51) are constant in time and are identical to the physical angles between the planes containing magnetic and velocity perturbations in physical space, see Figure 2, with

$$
\begin{aligned}
\tan \theta_{\mathrm{v} j} & =\frac{\delta v_{\phi}(z, t)}{\delta v_{r}(z, t)}=\text { const. } \\
\tan \theta_{\mathrm{b} j} & =\frac{\delta b_{\phi}(z, t)}{\delta b_{r}(z, t)}=\text { const. } .
\end{aligned}
$$

Finally, defining the angle $\theta_{\mathrm{bv} j}$ such that

$$
\theta_{\mathrm{bv} j}=\theta_{\mathrm{b} j}-\left(\theta_{\mathrm{v} j}+\frac{\pi}{2}\right),
$$

which implies that $\tan \theta_{\mathrm{b} j} \tan \left(\theta_{\mathrm{v} j}+\theta_{\mathrm{bv} j}\right)=-1$, and using the fact that

$$
\tan \left(\theta_{1}+\theta_{2}\right)=\frac{\tan \theta_{1}+\tan \theta_{2}}{1-\tan \theta_{1} \tan \theta_{2}}
$$



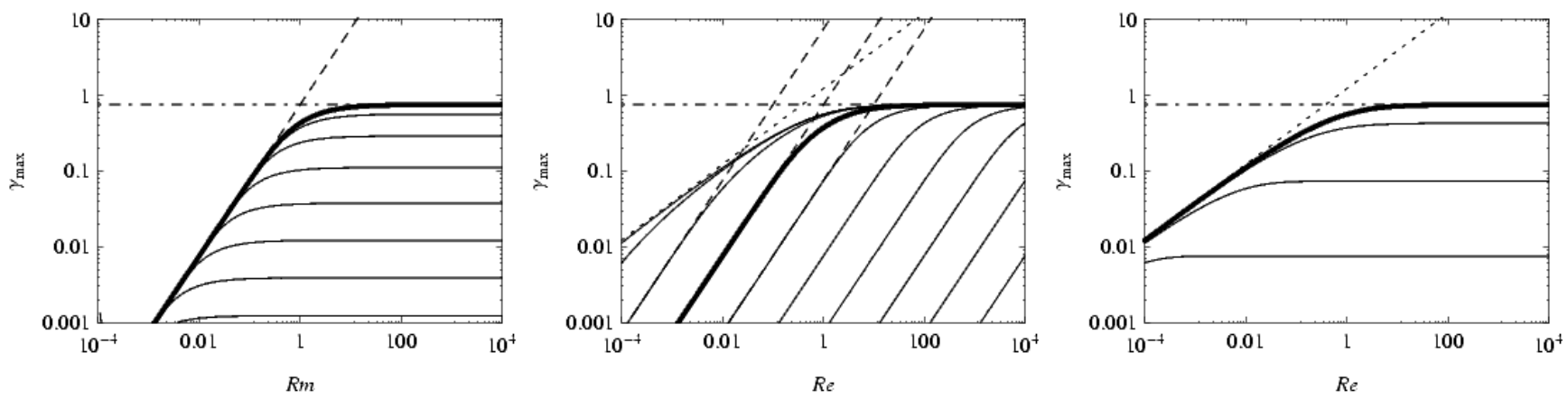

FIG. 5.- Maximum growth rate $\gamma_{\max }$ for Keplerian rotation in different dissipative regimes. The dot-dashed horizontal lines at $\gamma_{\mathrm{max}}=3 / 4$ represent the ideal MHD limit, eq. 82. Left: Maximum growth rate as a function of the magnetic Reynolds number for different values of the Reynolds number. The thick solid line denotes the inviscid limit, i.e., $\operatorname{Re} \rightarrow \infty$. The thin solid lines, in decreasing order, correspond to Re $=1,0.1, \ldots, 10^{-6}$. For magnetic Reynolds numbers larger than unity, the growth rate is independent of $\mathrm{Rm}$ regardless of the value of Re. The dashed line, calculated according to eq. 91, provides the correct asymptotic limit $\gamma_{\max } \propto$ Rm for small magnetic Reynolds numbers. Middle: Maximum growth rate $\gamma_{\max }$ as a function of the Reynolds number for different values of the magnetic Prandtl number. From left to right, the curves correspond to Pm $=10^{3}, 10^{2}, \ldots, 1$ (thick solid line), . ., 10 -6 . The dashed lines $\gamma_{\max } \propto \mathrm{RePm}=\mathrm{Rm}$ are calculated according to eq. 91, which leads to the correct result even for Pm $\gtrsim 1$ provided that the Reynolds number is sufficiently small. The dotted line $\gamma_{\max } \propto \operatorname{Re}^{1 / 2}$ results from eq. 102). Right: Maximum growth rate $\gamma_{\max }$ as a function of the Reynolds number for different values of the magnetic Reynolds number. The thick solid line corresponds to the ideal conductor limit, i.e., Rm $\rightarrow \infty$. The thin solid lines, in decreasing order, correspond to $\mathrm{Rm}=1,0.1,0.01$. For Reynolds numbers larger than unity, the growth rate is independent of Re regardless of the value of Rm. The dotted line, calculated according to eq. 102, provides the correct asymptotic limit $\gamma_{\max } \propto \mathrm{Re}^{1 / 2}$ for small Reynolds number.

it is not difficult to show that

$$
\tan \theta_{\mathrm{bv} j}=-\mu=-\left(\frac{\nu-\eta}{2}\right) k^{2} .
$$

This means that $\mu \neq 0$ provides a measure of how nonorthogonal velocity and magnetic field perturbations are.

It is evident that when the magnetic Prandtl number approaches unity viscous, resistive, MRI-driven magnetic and velocity perturbations tend to be orthogonal, i.e., $\tan \theta_{\mathrm{v} j} \tan \theta_{\mathrm{b} j}=-1$, and therefore

$$
\theta_{\mathrm{diff}} \equiv \theta_{\mathrm{b} j}-\theta_{\mathrm{v} j}=\frac{\pi}{2} \quad \text { for } \quad \operatorname{Pm}=1
$$

for every wavenumber $k$. This is illustrated in Figure 2 which shows the evolution of the angles $\theta_{\mathrm{b}}$ and $\theta_{\mathrm{v}}$ corresponding to the most unstable MRI mode as a function of the Reynolds/magnetic Reynolds number when the magnetic Prandtl number is equal to unity.

\section{PHYSICAL STRUCTURE OF MRI MODES}

The evolution of the physical structure of a single growing MRI mode with wavenumber $0<k<k_{\mathrm{c}}$ is characterized by its growth rate $\gamma_{+}$, the relative magnitude between the amplitudes of magnetic and velocity field perturbations, $b_{0} / v_{0}$, and the two angles defining the planes containing them, $\theta_{\mathrm{b}}$ and $\theta_{\mathrm{v}}$. For any reasonable spectrum of initial perturbations the mode that exhibits the fastest exponential growth, $\gamma_{\max }$, which we refer to as $k_{\max }$, will dominate the dynamics of the late time evolution of the viscous, resistive MRI. It is therefore of particular interest to characterize the physical properties of this fastest growing mode in different dissipative regimes.

\subsection{Marginal and Fastest Growing MRI-modes}

Because the eigenvalue associated with the unstable growing mode, $\gamma_{+}$, is always real for any combination of the Reynolds and magnetic Reynolds numbers, it is possible to find the marginally stable mode $k_{\mathrm{c}}$ such that $\gamma_{+}\left(k_{\mathrm{c}}\right) \equiv 0$. Setting $\sigma=0$ in equation 25), we obtain a polynomial in $k_{\mathrm{c}}$

$$
k_{\mathrm{c}}^{2}\left(1+\nu \eta k_{\mathrm{c}}^{2}\right)^{2}+\kappa^{2}\left(1+\eta^{2} k_{\mathrm{c}}^{2}\right)-4=0,
$$

valid for any value of the viscosity and resistivity. Note that $k_{\mathrm{c}}$ sets the minimum domain height for numerical simulations of viscous, resistive MRI-driven turbulence. Figure 3 shows the solutions of equation (70) in various dissipative regimes for Keplerian rotation. The analytic solutions of equation (70) are algebraically complicated but their asymptotic limits are rather simple. We find expressions for this critical wavenumber in several regimes of interest below.

In the ideal MHD limit, it is straightforward to find simple analytical expressions for the most unstable wavenumber, $k_{\max }$, and its associated growth rate, $\gamma_{\max }$. However, the analytical expressions that we derived for the eigenfrequencies in the non-ideal case, equation (46), are not amenable to the usual extremization procedure. More precisely, it is very challenging to find the values of $k_{\max }$ and $\gamma_{\max }$ that satisfy

$$
\left.\frac{d \gamma_{+}}{d k}\right|_{k_{\max }}=0
$$

Figures 4 and 5 show the solutions of this equation in various dissipative regimes.

Another possible path to find the values of the wavenumber $k_{\max }$, and the associated growth rate, is to use the fact that $\gamma_{\max }$ satisfies simultaneously the dispersion relation (25) and its derivative to eliminate $k_{\max }$ between these two and obtain a polynomial in $\gamma_{\max }$. The largest of the roots of this polynomial is the desired maximum growth rate. It is possible to find $k_{\max }$ following a similar methodology, but eliminating between the two polynomials $\gamma_{\max }$ instead. However, for arbitrary values of the viscosity and resistivity, both procedures lead to a seventh degree polynomial whose roots must be found numerically, defeating altogether the attempt to find analytical expressions for $k_{\max }$ and $\gamma_{\max }$.

Using as a guide the results shown in Figures 4 and 5, we follow an alternative procedure. The goal is to find simple analytical expressions to describe the asymptotic behavior of the most unstable mode, $k_{\max }$, and the maximum growth rate, $\gamma_{\max }$, in different dissipative regimes. It is evident from Figure 1 that $k_{\max }<1$ and $\gamma_{\max }<1$ for all the non-ideal MRI modes. This information can be used to simplify the disper- 
sion relation and its derivative so as to decrease their order without loosing vital information. This makes it possible to obtain manageable, but accurate, expressions for $k_{\max }$ and $\gamma_{\max }$ in different limiting regimes.

Figure 6 shows contour plots for the critical wavenumber, $k_{\mathrm{c}}$, the most unstable wavenumber, $k_{\max }$, and the maximum growth rate, $\gamma_{\max }$, as a function of the Reynolds and magnetic Reynolds numbers for Keplerian rotation. In all three panels, lighter gray areas correspond to larger values of $k_{\mathrm{c}}, k_{\max }$, and $\gamma_{\max }$, respectively. Note that in all the cases, the functional form of the contours naturally divides the plane (Re, Rm) in three distinctive regions that we denote according to I (ideal), $\mathrm{R}$ (resistive), and $\mathrm{V}$ (viscous). Note that when the most unstable wavenumber, $k_{\max }$, and the maximum growth rate, $\gamma_{\max }$, are considered, these regions can be associated with the regions where Re, Rm $\gg 1, \mathrm{Re} \gg \mathrm{Rm}$, and $\mathrm{Re} \ll \mathrm{Rm}$, respectively. The overlap between these regions is not as clear when the critical wavenumber $k_{\mathrm{c}}$ is considered and some care is needed when deriving approximated expressions for it.

\subsection{Ideal MRI Modes}

Let us first demonstrate briefly how the formalism presented in $\S 3$ reduces to previously known results in the ideal MHD limit. In the absence of dissipation, the eigenvalues $\left\{\sigma_{0, j}\right\}$, with $j=1,2,3,4$, are the roots of the dispersion relation associated with the ideal MRI (Balbus \& Hawley 1991, 1998),

$$
\left(k^{2}+\sigma_{0, j}^{2}\right)^{2}+\kappa^{2}\left(k^{2}+\sigma_{0, j}^{2}\right)-4 k^{2}=0,
$$

and are given by (Pessah, Chan, \& Psaltis 2006)

$$
\sigma_{0, j}= \pm\left(-\Lambda_{0} \pm \sqrt{\Delta_{0}}\right)^{1 / 2}
$$

where we have defined the quantities $\Lambda_{0}$ and $\Delta_{0}$ such that

$$
\begin{aligned}
& \Lambda_{0} \equiv \frac{\kappa^{2}}{2}+k^{2}, \\
& \Delta_{0} \equiv \frac{\kappa^{4}}{4}+4 k^{2} .
\end{aligned}
$$

The critical wavenumber for the onset of the ideal MRI is obtained by setting $\sigma_{0}=0$ in the dispersion relation (72), this leads to

$$
k_{\mathrm{c}}=\sqrt{2 q}=\sqrt{4-\kappa^{2}} .
$$

For all the modes with wavenumbers $k<k_{\mathrm{c}}$ the difference $\sqrt{\Delta_{0}}-\Lambda_{0}$ is positive and we can define the "growth rate" $\gamma_{0}$ and the "oscillation frequency" $\omega_{0}$ by

$$
\begin{aligned}
& \gamma_{0} \equiv\left(\sqrt{\Delta_{0}}-\Lambda_{0}\right)^{1 / 2}, \\
& \omega_{0} \equiv\left(\sqrt{\Delta_{0}}+\Lambda_{0}\right)^{1 / 2},
\end{aligned}
$$

both of which are real and positive (for all positive values of the parameter $q$ ). This shows that two of the solutions of equation (72) are real and the other two are imaginary. We can thus write the four eigenvalues in compact notation as

$$
\sigma_{0,1}=\gamma_{0}, \quad \sigma_{0,2}=-\gamma_{0}, \quad \sigma_{0,3}=i \omega_{0}, \quad \sigma_{0,4}=-i \omega_{0} .
$$

In the ideal MHD limit, it is evident that the velocity and magnetic field perturbations are orthogonal for any mode, i.e., $\tan \theta_{\mathrm{v}} \tan \theta_{\mathrm{b}}=-1$, see equation (68), and therefore

$$
\theta_{\mathrm{b}}=\theta_{\mathrm{v}}+\frac{\pi}{2} \text {. }
$$

The temporal evolution of a single MRI-unstable mode in physical space reduces to

$$
\boldsymbol{\delta}(z, t)=\frac{\sqrt{2} e^{\gamma_{0} t}}{\sqrt{v_{0}^{2}+b_{0}^{2}}}\left[\begin{array}{r}
v_{0} \cos \theta_{\mathrm{v}} \sin (k z) \\
v_{0} \sin \theta_{\mathrm{v}} \sin (k z) \\
b_{0} \sin \theta_{\mathrm{v}} \cos (k z) \\
-b_{0} \cos \theta_{\mathrm{v}} \cos (k z)
\end{array}\right] .
$$

These are essentially the (normalized) perturbations found in equation (4) in Goodman \& Xu (1994) ${ }^{6}$.

From the definition of the angle $\theta_{\mathrm{v}}$, see equation (50), it can be seen that

The maximum growth rate can be obtained by noting that $\gamma_{0}=q \sin \theta_{\mathrm{v}} \cos \theta_{\mathrm{v}}$ and therefore the maximum growth corresponds to

$$
\gamma_{\max }=\frac{q}{2}=1-\frac{\kappa^{2}}{4} .
$$

It then follows that, in the absence of dissipation, the planes containing the exponentially growing velocity and magnetic field perturbations are characterized by the angles

$$
\begin{aligned}
& \theta_{\mathrm{v}}=\frac{\pi}{4}, \\
& \theta_{\mathrm{b}}=\frac{3 \pi}{4},
\end{aligned}
$$

regardless of the value of the shearing parameter/epicyclic frequency.

Finally, noting that the wavenumber for which the maximum growth rate is realized is

$$
k_{\max }=\sqrt{1-\frac{\kappa^{4}}{16}},
$$

and using equation (54) for the ratio between the amplitudes of the magnetic and velocity fields we obtain

$$
\frac{b_{0}}{v_{0}}=\sqrt{\frac{4+\kappa^{2}}{4-\kappa^{2}}} .
$$

In $\S 6.2$ we derive equations for the MRI-driven Reynolds and Maxwell stresses, as well as the kinetic and magnetic energy densities associated with the perturbations. Equations (121), 122), (129), and (130), show why, in the ideal MHD limit, equations (83), (84), and (86) are the reason for which the ratio between the Maxwell to the Reynolds stresses is identical to the ratio between magnetic and kinetic energy densities for any shear parameter and equal to $5 / 3$ in the Keplerian case (Pessah, Chan, \& Psaltis 2006).

\subsection{MRI Modes with $\mathrm{Re} \gg \mathrm{Rm}$}

\footnotetext{
${ }^{6}$ Note that the angle $\gamma$ in Goodman \& Xu (1994), in our notation defined by $\tan \gamma=-\delta B_{r} / \delta B_{\phi}$, is such that $\gamma=0$ in the positive azimuthal axis and it takes increasingly positive values in the counter-clockwise direction.
} 

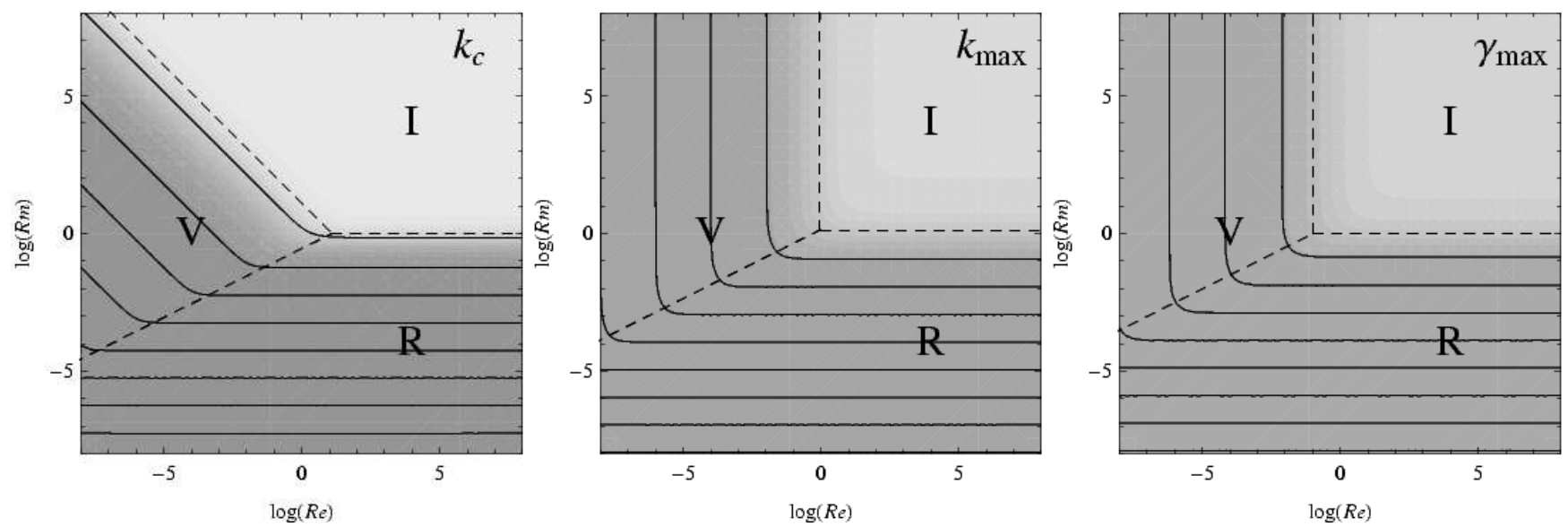

FIG. 6.- Contour plots for the critical wavenumber, $k_{\mathrm{c}}$, the most unstable wavenumber, $k_{\max }$, and the maximum growth rate, $\gamma_{\max }$, for Keplerian rotation. In all three panels, lighter gray areas correspond to larger values of $k_{\mathrm{c}}, k_{\max }$, and $\gamma_{\max }$, respectively. The solid lines highlight the contours for $k_{\mathrm{c}}=1, \ldots, 10^{-7}$ and $k_{\max }=10^{-1}, \ldots, 10^{-8}$. The labels I (ideal), $\mathrm{R}$ (resistive), and $\mathrm{V}$ (viscous), denote the three regions of the (Re, $\left.\mathrm{Rm}\right)$ plane where equations (76), 85], and (82); (87), 90) and (91); and 298, [101), and (102) are valid, respectively. The dashed lines dividing the three regions are obtained by equating neighboring approximations for $k_{\mathrm{c}}, k_{\max }$, and $\gamma_{\max }$.
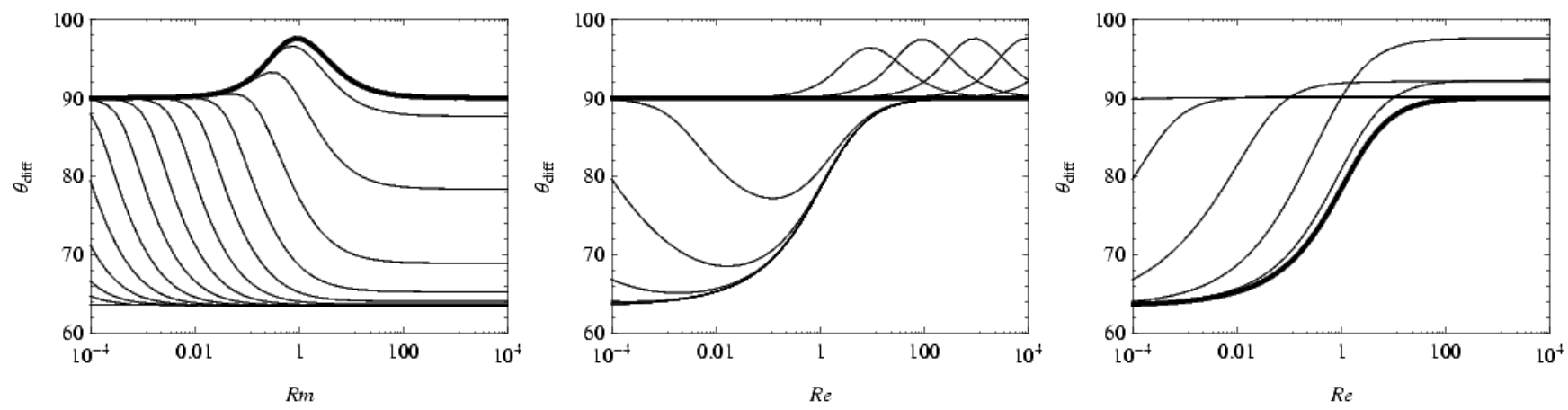

FIG. 7.- Opening angle, $\theta_{\text {diff }}=\theta_{\mathrm{b}}-\theta_{\mathrm{v}}$, between the planes containing the fastest exponentially growing magnetic and velocity perturbations for Keplerian rotation in various dissipative regimes. In the ideal MHD limit the opening angle is $\theta_{\text {diff }}=\pi / 2$, see eqs. 83. and 84]. Left: opening angle $\theta_{\text {diff }}$ as a function of the magnetic Reynolds number for different values of the Reynolds number. The thick solid line denotes the inviscid limit, i.e., Re $\rightarrow \infty$. The thin solid

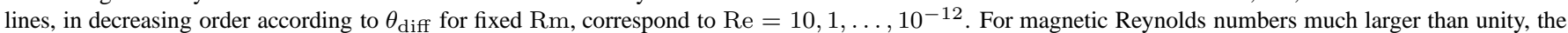
opening angle is independent of Rm regardless of the value of Re. For sufficiently small/large Rm, $\theta_{\text {diff }} \rightarrow \pi / 2$ provided that Re $\gg$ Rm. For sufficiently small/large $\mathrm{Rm}, \theta_{\text {diff }} \rightarrow \pi / 2-\arctan (\kappa / 2)$ provided that $\mathrm{Rm} \ll \operatorname{Re}$. Note that this corresponds to $\theta_{\text {diff }}=63^{\circ} 26^{\prime}$ for a Keplerian disk. The only conditions under which $\theta_{\text {diff }}$ exceeds $\pi / 2$ are such that $\operatorname{Re}>\operatorname{Rm} \simeq 1$. Middle: opening angle $\theta_{\text {diff }}$ as a function of the Reynolds number for different values of the magnetic Prandtl number. The thick solid line at $\theta_{\text {diff }}=\pi / 2$ corresponds to $\mathrm{Pm}=1$, see eq. 69) and Fig. 22 The thin solid lines with peaks at $\theta_{\text {diff }}>\pi / 2$ correspond, from left to right, to $\mathrm{Pm}=10^{-1}, 10^{-2}, \ldots$ The thin solid lines, with $\theta_{\text {diff }}<\pi / 2$, in decreasing order according to $\theta_{\text {diff }}$ for fixed Re, correspond to $\mathrm{Pm}=10,10^{2}, \ldots$. Note that for small Re, $\theta_{\text {diff }} \rightarrow \pi / 2$ for $\mathrm{Pm} \simeq 1$, while $\theta_{\text {diff }} \rightarrow \pi / 2-\arctan (\kappa / 2)$ for Pm $\gg 1$. Right: opening angle $\theta_{\text {diff }}$ as a function of the Reynolds number for different values of the magnetic Reynolds number. The thick solid line corresponds to the ideal conductor limit, i.e., $\mathrm{Rm} \rightarrow \infty$. The thin solid lines, from right to left, correspond to $\mathrm{Rm}=10, \ldots, 10^{-3}$. For Reynolds numbers larger than unity, the opening angle is independent of Re regardless of the value of $\mathrm{Rm}$.

Lets us consider the inviscid, poorly conducting limit described by $\nu=0$ and $\eta \gg 1$. In this case, the marginally stable mode satisfying equation (70) is given by

$$
k_{\mathrm{c}}=\sqrt{\frac{4-\kappa^{2}}{1+\eta^{2} \kappa^{2}}} .
$$

The dependence of this critical wavenumber on the magnetic Reynolds number is shown on the left panel in Figure 3, which shows that for small magnetic Reynolds numbers $k_{\mathrm{c}} \propto R m$.

As discussed in $\S$ 4.1, finding an analytic expression for the maximum growth rate and wavenumber associated with it is not as straightforward. The left panel of Figure 5 suggests that in the limit $\operatorname{Re} \rightarrow \infty$ and $\mathrm{Rm} \ll 1$, the maximum growth rate is linear in the magnetic Reynolds number, $\gamma_{\max } \propto \mathrm{Rm} \propto \eta^{-1}$. This information can be used to derive asymptotic expressions for the dispersion relation (25) and its derivative. The leading order contributions are given by

$\kappa^{2} \gamma_{\max }^{2}+2 \kappa^{2} \eta k_{\max }^{2} \gamma_{\max }+\kappa^{2} \eta^{2} k_{\max }^{4}+\left(\kappa^{2}-4\right) k_{\max }^{2}=0$

and

$$
2 \kappa^{2} \eta \gamma_{\max }+2 \kappa^{2} \eta^{2} k_{\max }^{2}+\kappa^{2}-4=0,
$$

respectively.

Eliminating either $\gamma_{\max }$ or $k_{\max }$ between equations and (89) we obtain

$$
k_{\max }=\frac{1}{\eta} \sqrt{\frac{4-\kappa^{2}}{4 \kappa^{2}}},
$$

and

$$
\gamma_{\max }=\frac{1}{\eta} \frac{4-\kappa^{2}}{4 \kappa^{2}}
$$



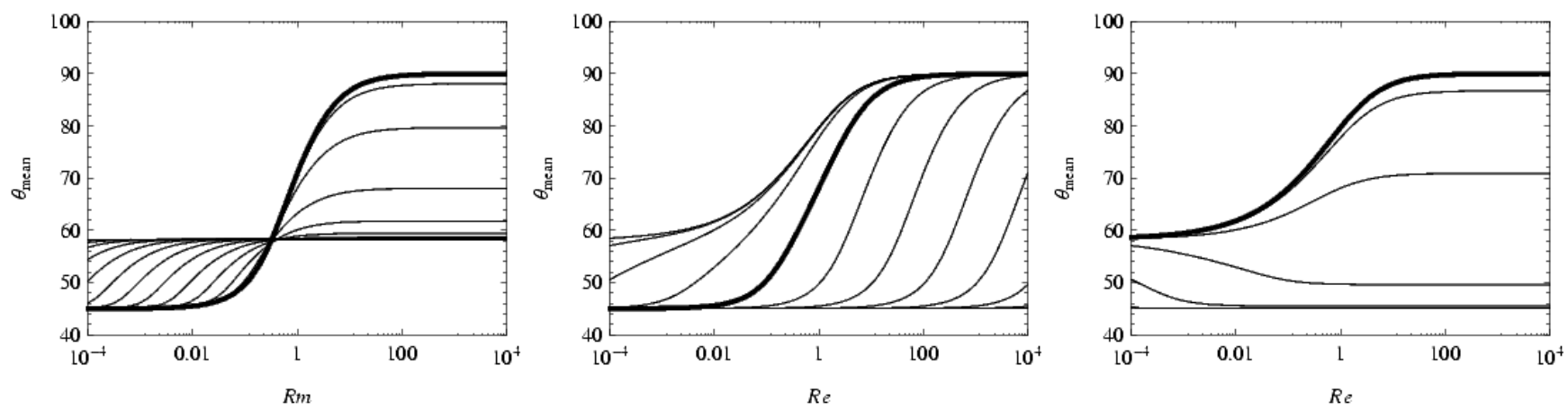

FIG. 8.- Mean angle, $\theta_{\text {mean }}=\left(\theta_{\mathrm{v}}+\theta_{\mathrm{b}}\right) / 2$, defined by the fastest exponentially growing velocity and magnetic perturbations for Keplerian rotation in various dissipative regimes. Note that $\theta_{\text {mean }}=\pi / 2$ in the ideal MHD limit, see eqs. 83) and 84]. Left: mean angle $\theta_{\text {mean }}$ as a function of the magnetic Reynolds number for different values of the Reynolds number. The thick solid line denotes the inviscid limit, i.e., Re $\rightarrow \infty$. There is a critical magnetic Reynolds number $\mathrm{Rm}_{\mathrm{C}} \lesssim 1$ that differentiates the asymptotic limits of $\theta_{\text {mean }}$ for large and small Reynolds numbers. When the Reynolds number changes from Re $\ll R m$ to $\operatorname{Re} \gg \mathrm{Rm}$, the mean angle evolves according to $\theta_{\text {mean }}:[\pi / 2+\arctan (\kappa / 2)] / 2 \rightarrow \pi / 2$ for $\mathrm{Rm}>\mathrm{Rm}_{\mathrm{c}}$, while $\theta_{\text {mean }}:[\pi / 2+\arctan (\kappa / 2)] / 2 \rightarrow \pi / 4$ for $\mathrm{Rm}<\mathrm{Rm}_{\mathrm{c}}$. Note that for Keplerian rotation $[\pi / 2+\arctan (\kappa / 2)] / 2=58^{\circ} 17^{\prime}$. Middle: opening angle $\theta_{\text {diff }}$ as a function of the Reynolds number for different values of the magnetic Prandtl number. The thick solid line corresponds to $\mathrm{Pm}=1$. The thin solid lines to the right correspond to smaller values $\mathrm{Pm}=10^{-1}, 10^{-2}, \ldots$. The thin solid lines to the left correspond to $\mathrm{Pm}=10,10^{2}, \ldots$. Note that for small Re, $\theta_{\text {mean }} \rightarrow \pi / 4$ for Pm $\langle 1$, while $\theta_{\text {mean }} \rightarrow[\pi / 2+\arctan (\kappa / 2)] / 2$ for Pm $\gg 1$. Right: mean angle $\theta_{\text {mean }}$ as a function of the magnetic Reynolds number for different values of the Reynolds number. The thick solid line corresponds to the ideal conductor limit, i.e., $\mathrm{Rm} \rightarrow \infty$. The thin solid lines, in decreasing order, correspond to Re $=10,1, \ldots$. For Reynolds numbers larger than unity, the mean angle is independent of Re regardless of the value of Rm.

In this case, $\gamma_{\max }=\eta k_{\max }^{2}$ for any value of the epicyclic frequency $\kappa$.

The dependence of both $k_{\max }$ and $\gamma_{\max }$ in this limiting case is shown with dashed lines in the left panels of Figures 4 and 5. respectively. The agreement between equations (90) and (91) and the solutions to the full dispersion relation (25) in the case $\nu=0$ and $\eta \gg 1$ is excellent, only breaking down close to magnetic Reynolds numbers of order unity. Note that even though the equations (90) and (91) were derived under the assumption of an inviscid fluid, i.e., $\nu=0$, these expressions can describe the asymptotic behavior of both $k_{\max }$ and $\gamma_{\max }$ for finite Reynolds numbers provided that the conditions $\mathrm{Re} \gg \mathrm{Rm}$ and $\mathrm{Rm} \ll 1$ are satisfied.

Substituting the asymptotic expressions for $k_{\max }$ and $\gamma_{\max }$ in equations (90) and (91) into equation (54) we obtain the ratio between the amplitudes of the magnetic and velocity field perturbations

$$
\frac{b_{0}}{v_{0}}=\frac{\eta \kappa^{3}}{\sqrt{4-\kappa^{2}}} .
$$

Therefore, inviscid, resistive MRI-unstable modes are dominated by magnetic field perturbations. Note that the ratio between amplitudes increases linearly with resistivity.

The asymptotic behavior for the angles characterizing velocity and magnetic field perturbations, equations (50) and (51), are given by

$$
\tan \theta_{\mathrm{v}}=\frac{1}{2 \kappa^{2} \eta}
$$

and

$$
\tan \theta_{\mathrm{b}}=-\eta \kappa^{2} .
$$

In the limit $\mathrm{Re} \rightarrow \infty$ and $\mathrm{Rm} \rightarrow 0$, we obtain

$$
\begin{aligned}
& \lim _{\eta \rightarrow \infty} \theta_{\mathrm{v}}=0, \\
& \lim _{\eta \rightarrow \infty} \theta_{\mathrm{b}}=\frac{\pi}{2} .
\end{aligned}
$$

We therefore conclude that in the regime of large Reynolds numbers and small magnetic Reynolds numbers, magnetic field perturbations are larger than velocity field perturbations, both fields tend to be orthogonal and aligned with the azimuthal and radial directions, respectively, see Figures 7, 8, and 9 .

\subsection{MRI Modes with $\mathrm{Re}=\mathrm{Rm} \ll 1$}

When the magnetic Prandtl number is unity, and with $\eta=$ $\nu \gg 1$, the marginally stable mode satisfying equation 70 is given by

$$
k_{\mathrm{c}}=\frac{\sqrt{4-\kappa^{2}}}{\eta \kappa} .
$$

The dependence of this critical wavenumber on the Reynolds number is shown in the middle panel in Figure 3 . Incidentally, equation 97 corresponds to the asymptotic limit $\eta \gg 1$ of equation 87 .

It is not hard to see that the leading order contributions to the dispersion relation and its derivative in the limit $\mathrm{Re}=$ $\mathrm{Rm} \ll 1$ are identical to the ones obtained in the case $\mathrm{Re} \rightarrow$ $\infty$ and $\mathrm{Rm} \ll 1$. Therefore, all the expressions for $k_{\max }$, $\gamma_{\max }, \theta_{\mathrm{v}}$, and $\theta_{\mathrm{b}}$, derived in $\S 4.3$ are also valid in this case. The dependence of both $k_{\max }$ and $\gamma_{\max }$ in this limiting case is shown with dashed lines in the middle panels of Figures 4 and 5. respectively. The agreement between equations (101) and (102) and the solutions to the full dispersion relation 25 in the case $\nu=\eta$, i.e., $\mathrm{Pm}=1$, is also excellent in this case.

\subsection{MRI Modes with $\mathrm{Re} \ll \mathrm{Rm}$}

Lets us consider next the highly viscous, ideal conductor limit described by $\nu \gg 1$ and $\eta=0$. In this case, the marginally stable mode satisfying equation (70) is given by

$$
k_{\mathrm{c}}=\left(\frac{\sqrt{4-\kappa^{2}}}{\nu \eta}\right)^{1 / 3} \text {. }
$$

The right panel of Figure 5 suggests that in the limit Re $\ll$ 1 and $\mathrm{Rm} \rightarrow \infty$, the dependence of the maximum growth 


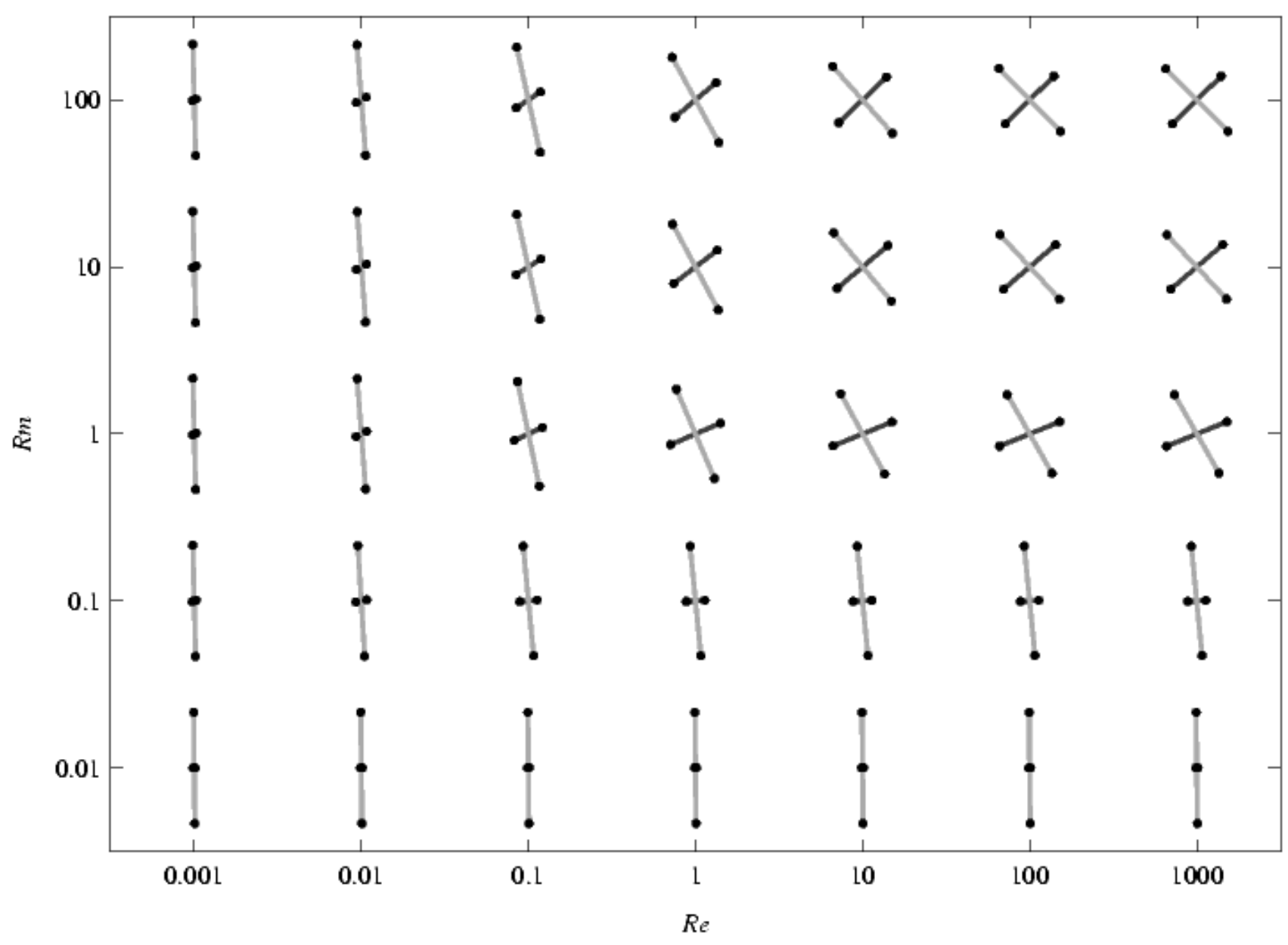

FIG. 9.- Geometrical representation of viscous, resistive MRI modes for varying Reynolds and magnetic Reynolds numbers. The black/gray lines denote velocity/magnetic components of the most unstable mode.

on the Reynolds number is $\gamma_{\max } \propto \operatorname{Re}^{1 / 2} \propto \nu^{-1 / 2}$. This information can be used to derive asymptotic expressions for the dispersion relation (25) and its derivative. The leading order contributions are given by

$$
\left(\kappa^{2}+\nu^{2} k_{\max }^{4}\right) \gamma_{\max }^{2}+\left(\kappa^{2}-4\right) k_{\max }^{2}=0,
$$

and

$$
2 \nu^{2} k_{\max }^{2} \gamma_{\max }^{2}+\kappa^{2}-4=0
$$

respectively.

Eliminating either $\gamma_{\max }$ or $k_{\max }$ between these equations we obtain

$$
k_{\max }=\sqrt{\frac{\kappa}{\nu}},
$$

and

$$
\gamma_{\max }=\sqrt{\frac{4-\kappa^{2}}{2 \nu \kappa}} .
$$

Eliminating the epicyclic frequency between equations 102 and (101) we obtain

$$
\gamma_{\max }^{2}=\frac{4-\nu^{2} k_{\max }^{4}}{2 \nu^{2} k_{\max }^{2}} .
$$

The dependence of both $k_{\max }$ and $\gamma_{\max }$ in this limiting case is shown with dashed lines in the right panels of Figures 4 and 5 respectively. The agreement between equations (101) and (102) and the solutions to the full dispersion relation 25 in the case $\nu \gg 1$ and $\eta=0$ is excellent, only breaking down close to Reynolds numbers of order unity. Note that even though the equations (101) and (102) were derived under the assumption of a perfectly conducting fluid, i.e., $\eta=0$, these expressions can describe the asymptotic behavior of both $k_{\max }$ and $\gamma_{\max }$ for finite Reynolds numbers provided that the conditions $\mathrm{Re} \ll \mathrm{Rm}$ and $\mathrm{Re} \ll 1$ are satisfied.

Substituting the asymptotic expressions for $k_{\max }$ and $\gamma_{\max }$ in equations (101) and (102) into equation (54) we obtain the relative amplitude of the magnetic and velocity field perturbations

$$
\frac{b_{0}}{v_{0}}=2 \sqrt{\frac{\nu \kappa^{3}}{4+\kappa^{2}}} .
$$

Therefore, viscous, conducting MRI-unstable modes are also dominated by magnetic field perturbations. In this case, the ratio between amplitudes increases only with the square root of the viscosity.

The asymptotic behavior for the angles characterizing velocity and magnetic field perturbations, equations (50) and 
51), are given by

$$
\tan \theta_{\mathrm{v}}=\frac{\kappa}{2}+\frac{4+\kappa^{2}}{\sqrt{2 \nu \kappa\left(4-\kappa^{2}\right)}},
$$

and

$$
\tan \theta_{\mathrm{b}}=\sqrt{\frac{\nu\left(4-\kappa^{2}\right)}{2 \kappa}} .
$$

In the limit $\operatorname{Re} \rightarrow 0$ and $\mathrm{Rm} \rightarrow \infty$, we obtain

$$
\begin{aligned}
\lim _{\nu \rightarrow \infty} \theta_{\mathrm{v}} & =\arctan \left(\frac{\kappa}{2}\right), \\
\lim _{\nu \rightarrow \infty} \theta_{\mathrm{b}} & =\frac{\pi}{2} .
\end{aligned}
$$

For Keplerian rotation, the angle between the fastest growing velocity field perturbation and the radial direction is given by $\theta_{\mathrm{v}}=\arctan (1 / 2)=26^{\circ} 34^{\prime}$. Therefore, the opening angle between the planes containing velocity and magnetic field perturbations is $\theta_{\text {diff }}=\theta_{\mathrm{b}}-\theta_{\mathrm{v}}=63^{\circ} 26^{\prime}$ and their mean value is $\theta_{\text {mean }}=\left(\theta_{\mathrm{b}}+\theta_{\mathrm{v}}\right) / 2=58^{\circ} 17^{\prime}$. The right panels of Figures 7 and 8 show that both of these results are in perfect agreement with the asymptotic behavior of the full solutions derived directly from the original dispersion relation (25).

We therefore conclude that in the regime of small Reynolds numbers and large magnetic Reynolds numbers, magnetic perturbations are larger than velocity perturbations. In this case, however, the perturbed magnetic and velocity fields are not orthogonal. The perturbed magnetic field tends to be aligned with the azimuthal direction but the velocity field perturbations do not tend to be aligned with the radial direction. The angle between both fields is determined entirely by the epicyclic frequency $\kappa$, see Figures 7, 8, and 9

\subsection{MRI Modes with $\mathrm{Re}=\mathrm{Rm} \gg 1$}

As we show in Appendix A when the Reynolds and magnetic Reynolds numbers are large enough the solutions to the dispersion relation (25) tend smoothly to the solutions of the dispersion relation (72) for the idealized case. Therefore, in this limit we recover all the expressions derived in $\S 4.2$.

\section{PHYSICS OF MAXIMALLY UNSTABLE MRI MODES}

We have shown that the expressions derived for the most unstable wavenumber, $k_{\max }$, and its associated growth rate, $\gamma_{\max }$, obtained from the simplified equations (88) and (99) are good approximations to the solutions obtained directly from the dispersion relation (25), in the limits $R e \gg R m$ and $R e \ll$ $\mathrm{Rm}$, respectively. We can now identify the various terms in the original set of equations of motion (7)-(10) that lead to equations (88) and (99). This allows us to better understand the forces that act to destabilize magnetized fluid elements.

For the sake of clarity we write the equations in this section with physical dimensions. We represent the temporal derivatives with a dot and the derivatives with respect to the vertical coordinate $z$ with a prime.

\subsection{MRI Modes with $\mathrm{Re} \gg \mathrm{Rm}$}

The equations of motion that lead to the dispersion relation (88) are given by

$$
\begin{aligned}
\delta \dot{v}_{r} & =2 \Omega_{0} \delta v_{\phi}+\frac{\bar{B}_{z}}{4 \pi \rho} \delta B_{r}^{\prime}, \\
0 & =-(2-q) \Omega_{0} \delta v_{r}+\frac{\bar{B}_{z}}{4 \pi \rho} \delta B_{\phi}^{\prime}, \\
\delta \dot{B}_{r} & =\bar{B}_{z} \delta v_{r}^{\prime}+\eta \delta B_{r}^{\prime \prime}, \\
\delta \dot{B}_{\phi} & =-q \Omega_{0} \delta B_{r}+\eta \delta B_{\phi}^{\prime \prime} .
\end{aligned}
$$

Therefore, maximally unstable modes with $\mathrm{Re} \gg \mathrm{Rm}$ are characterized by motions with radial acceleration due to the Coriolis force acting on azimuthally displaced fluid elements and magnetic tension. Azimuthal force balance is attained via the joint action of the Coriolis force acting on radially displaced fluid elements, radial advection of background flow, and magnetic tension in the azimuthal direction. The rate of change of the radial magnetic field perturbations is due to the creation of radial field out of the vertical background frozen into the radial velocity field with a vertical gradient and field diffusion. The rate of change of the azimuthal magnetic field perturbations is due to the shearing of radial magnetic field perturbations and field diffusion.

\subsection{MRI Modes with $\mathrm{Re} \ll \mathrm{Rm}$}

The set of equations that lead to the dispersion relation (99) are given by

$$
\begin{aligned}
0 & =2 \Omega_{0} \delta v_{\phi}+\nu \delta v_{r}^{\prime \prime}, \\
0 & =-(2-q) \Omega_{0} \delta v_{r}+\frac{\bar{B}_{z}}{4 \pi \rho} \delta B_{\phi}^{\prime}+\nu \delta v_{\phi}^{\prime \prime}, \\
\delta \dot{B}_{r} & =\bar{B}_{z} \delta v_{r}^{\prime}, \\
\delta \dot{B}_{\phi} & =-q \Omega_{0} \delta B_{r} .
\end{aligned}
$$

In this case, maximally unstable modes with $\mathrm{Re} \ll \mathrm{Rm}$ are characterized by fluid displacements that take place under force balance in both the radial and azimuthal directions. The Coriolis force acting on azimuthally displaced fluid elements is balanced by the viscous force in the radial direction. Azimuthal force balance is attained via the joint action of the Coriolis force acting on radially displaced fluid elements, radial advection of background flow, magnetic tension, and the viscous force in the azimuthal direction. The rate of change of the radial magnetic field perturbations is due to the creation of radial field out of the vertical background frozen into the radial velocity field with a vertical gradient. Finally, the rate of change of the azimuthal magnetic field perturbations is due to the shearing of radial magnetic field perturbations.

\section{NON-IDEAL MRI-DRIVEN STRESSES AND ENERGY DENSITIES}

The $i, j$ components of the mean Reynolds and Maxwell stresses associated with the velocity and magnetic field perturbations are given by

$$
\begin{aligned}
\bar{R}_{i j}(t) & \equiv\left\langle\delta v_{i}(z, t) \delta v_{j}(z, t)\right\rangle, \\
\bar{M}_{i j}(t) & \equiv\left\langle\delta b_{i}(z, t) \delta b_{j}(z, t)\right\rangle,
\end{aligned}
$$

where the brackets denote mean values obtained via integration over the disk scale-height, $2 H$. These mean values can 

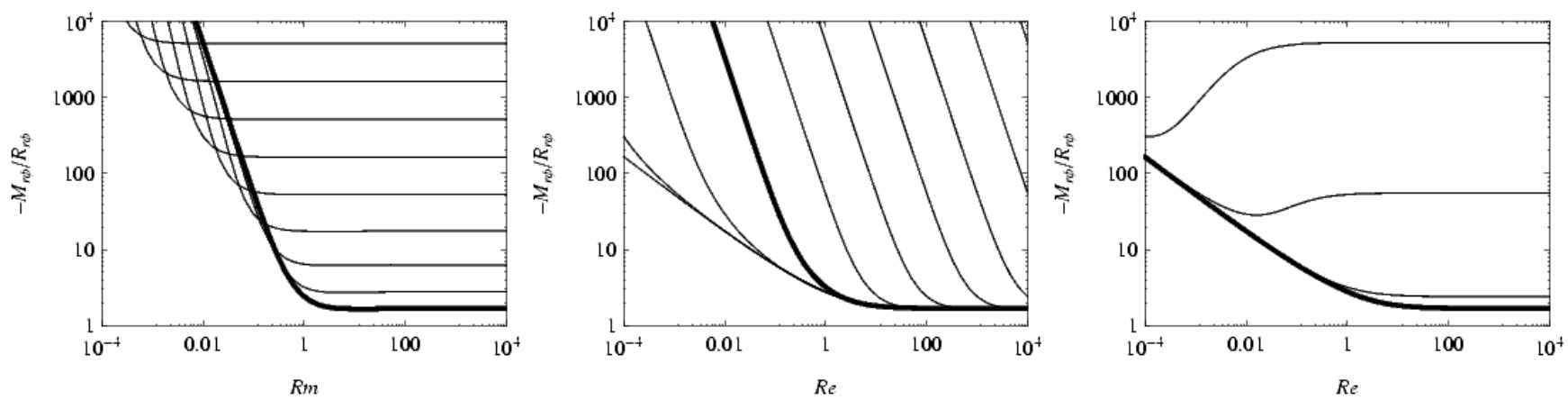

FIG. 10.- Ratio between the Maxwell and the Reynolds stresses, $-\bar{M}_{r \phi} / \bar{R}_{r \phi}$, for Keplerian rotation in different dissipative regimes. Left: Ratio between the Maxwell and the Reynolds stresses as a function of the magnetic Reynolds number for different values of the Reynolds number. The thick solid line denotes the inviscid limit, i.e., Re $\rightarrow \infty$. The thin solid lines, in increasing order according to their asymptotic value at high magnetic Reynolds numbers correspond to $\mathrm{Re}=1,0.1, \ldots$ For $\mathrm{Rm}>1$, the ratio is independent of $\mathrm{Rm}$ regardless of the value of Re. Middle: Ratio between the Maxwell and the Reynolds stresses as a function of the Reynolds number for different values of the magnetic Prandtl number. The various curves, from left to right, correspond to Pm $=10^{3}, 10^{2}, \ldots$ The thick solid line corresponds to the case $\mathrm{Pm}=1$. Right: Ratio between the Maxwell and the Reynolds stresses as a function of the Reynolds number for different values of the magnetic Reynolds number. The thick solid line corresponds to the ideal conductor limit, i.e., Rm $\rightarrow \infty$. The thin solid lines, in increasing order according to their asymptotic value at high Reynolds numbers correspond to $\mathrm{Rm}=1,0.1,0.01$. The Maxwell stress is larger than the Reynolds stress for any combination of the Reynolds and magnetic Reynolds numbers. The minimum value of this ratio is achieved in the ideal MHD regime, where $-\bar{M}_{r \phi} / \bar{R}_{r \phi}=5 / 3$, for Keplerian rotation.

also be calculated directly from the perturbations in Fourier space according to (Pessah, Chan, \& Psaltis 2006)

$$
\begin{gathered}
\bar{R}_{i j}(t) \equiv 2 \sum_{n=1}^{\infty} \mathcal{R} e\left[\hat{v}_{i}\left(k_{n}, t\right) \delta \hat{v}_{j}^{*}\left(k_{n}, t\right)\right] \\
\bar{M}_{i j}(t) \equiv 2 \sum_{n=1}^{\infty} \mathcal{R} e\left[\delta \hat{b}_{i}\left(k_{n}, t\right) \delta \hat{b}_{j}^{*}\left(k_{n}, t\right)\right]
\end{gathered}
$$

where $\mathcal{R} e[]$ stands for the real part of the quantity between square brackets. The off-diagonal components of these stresses relate to angular momentum transport while their traces relate to the kinetic and magnetic energy in the perturbations.

At late times, during the exponential growth of the instability, the branch of unstable modes will dominate the growth of the perturbations and the most important (secular) contribution to the mean stresses will be given by the most unstable mode. The leading order contribution to these stress components are thus obtained by considering the most unstable solutions to the set of equations (7)-(10), which are given by equation (63) when $k=k_{\max }$ with $\gamma_{+}=\gamma_{\max }$.

\subsection{Non-ideal MRI Stresses}

A measure of the angular momentum transport driven by the most unstable MRI modes and mediated by the correlated perturbations in the velocity and magnetic fields is obtained by setting $(i, j)=(r, \phi)$ in equations (117) and (118). To leading order in time we obtain

$$
\begin{aligned}
& \bar{R}_{r \phi}(t)=\frac{1}{2} \frac{v_{0}^{2} e^{2 \gamma_{\max } t}}{v_{0}^{2}+b_{0}^{2}} \sin \left(2 \theta_{\mathrm{v}}\right), \\
& \bar{M}_{r \phi}(t)=\frac{1}{2} \frac{b_{0}^{2} e^{2 \gamma_{\max } t}}{v_{0}^{2}+b_{0}^{2}} \sin \left(2 \theta_{\mathrm{b}}\right) .
\end{aligned}
$$

The results derived in $\S 4$, together with Figures 7,8 , and 9 . show that the angles $\theta_{\mathrm{v}}$ and $\theta_{\mathrm{b}}$ corresponding to the most unstable mode, $k_{\max }$, always satisfy

$$
0 \leq \theta_{\mathrm{v}} \leq \arctan \left(\frac{\kappa}{2}\right) \leq \frac{\pi}{4}
$$

$$
\frac{\pi}{2} \leq \theta_{\mathrm{b}} \leq \frac{3 \pi}{4}
$$

Both of these inequalities show explicitly that the mean Reynolds and Maxwell stresses will be, respectively, positive and negative,

$$
\bar{R}_{r \phi}(t)>0 \quad \text { and } \quad \bar{M}_{r \phi}(t)<0 .
$$

This, in turn, implies that the mean total MRI-driven stress will be always positive, i.e.,

$$
\bar{T}_{r \phi}(t)=\bar{R}_{r \phi}(t)-\bar{M}_{r \phi}(t)>0,
$$

driving a net outward flux of angular momentum for any combination of Reynolds and magnetic Reynolds numbers.

We conclude this section by calculating the ratio $-\bar{M}_{r \phi}(t) / \bar{R}_{r \phi}(t)$ at late times during the exponential growth of the instability. We obtain

$$
\lim _{t \gg 1} \frac{-\bar{M}_{r \phi}(t)}{\bar{R}_{r \phi}(t)}=-\frac{v_{0}^{2}}{b_{0}^{2}} \frac{\sin \left(2 \theta_{\mathrm{v}}\right)}{\sin \left(2 \theta_{\mathrm{b}}\right)} .
$$

Using the definitions for the ratio $v_{0} / b_{0}$ (eq. [54]) and the angles $\theta_{\mathrm{v}}$ and $\theta_{\mathrm{b}}$ (eqs. [50] and [51]), it can be seen that the magnitude of the Maxwell stress, $-\bar{M}_{r \phi}(t)$, is always larger than the magnitude of the Reynolds stress, $\bar{R}_{r \phi}(t)$, provided that the flow is Rayleigh-stable, i.e.,

$$
-\bar{M}_{r \phi}(t)>\bar{R}_{r \phi}(t) \text { for } 0<q<2 .
$$

Figure 10 shows the ratio between the Maxwell and the Reynolds stresses in various dissipative regimes for Keplerian rotation. Note that when the Reynolds and magnetic Reynolds numbers are large enough we recover the result $-\bar{M}_{r \phi} / \bar{R}_{r \phi}=5 / 3$, which coincides with the value of this ratio in the ideal MHD case (Pessah, Chan, \& Psaltis 2006).

\subsection{Non-ideal MRI Energetics}

The mean energy densities associated with the perturbations in the velocity and magnetic field are given by

$$
\begin{aligned}
& \bar{E}_{K}(t)=\frac{1}{2}\left(\bar{R}_{r r}+\bar{R}_{\phi \phi}\right), \\
& \bar{E}_{M}(t)=\frac{1}{2}\left(\bar{M}_{r r}+\bar{M}_{\phi \phi}\right) .
\end{aligned}
$$


Substituting the expressions for the most unstable MRI-driven perturbations from equation (63) into the definitions for the diagonal components of the Reynolds and Maxwell stresses, equations (117)-(118), respectively, we obtain

$$
\begin{aligned}
& \bar{E}_{K}(t)=\frac{1}{2} \frac{v_{0}^{2} e^{2 \gamma_{\max } t}}{v_{0}^{2}+b_{0}^{2}}, \\
& \bar{E}_{M}(t)=\frac{1}{2} \frac{b_{0}^{2} e^{2 \gamma_{\max } t}}{v_{0}^{2}+b_{0}^{2}} .
\end{aligned}
$$

Using the definitions for the ratio $v_{0} / b_{0}$, equation (54), it can be seen that for non-ideal MRI modes the mean energy associated with magnetic perturbations is always larger than the mean energy corresponding to velocity perturbations as long as the flow is Rayleigh-stable. Figure 11 shows the ratio between the magnetic and the kinetic energy densities in various dissipative regimes for Keplerian rotation.

In the limit of large Reynolds and magnetic Reynolds numbers, we recover the result $\bar{E}_{M} / \bar{E}_{K}=5 / 3$, which coincides with the value of this ratio in the ideal MHD case and also with the ratio between the magnitudes of the Maxwell and Reynolds stresses in the ideal case (Pessah, Chan, \& Psaltis 2006). Note, however, that for arbitrary Reynolds and magnetic Reynolds numbers, it is no longer true that the ratio between mean magnetic and mean kinetic energies is equal to the ratio between the magnitude of the mean Maxwell and the mean Reynolds stresses. This can be seen by comparing Figures 10 and 11

Finally, comparing equations (121) and (122) with (131) and (132), it immediately follows that

$$
\begin{aligned}
\bar{R}_{r \phi}(t) & \leq \bar{E}_{K}(t), \\
-\bar{M}_{r \phi}(t) & \leq \bar{E}_{M}(t) .
\end{aligned}
$$

This result, in turn, implies that the total mean energy associated with the perturbations, $\bar{E}(t)=\bar{E}_{K}(t)+\bar{E}_{M}(t)$, sets an upper bound on the total mean stress, i.e.,

$$
\bar{T}_{r \phi}(t) \leq \bar{E}(t),
$$

for any Reynolds and magnetic Reynolds numbers.

\section{SUMMARY \& DISCUSSION}

We investigated the effects of viscosity and resistivity on the stability of differentially rotating plasmas threaded by a magnetic field perpendicular to the shear. We have shown that the most powerful incompressible MRI modes are exact solutions of the MHD equations for arbitrary combinations of the Reynolds and magnetic Reynolds numbers. We have derived analytical expressions for the eigenfrequencies as well as for the eigenmodes describing the MRI in viscous, resistive media and provided a detailed description of the physical properties of these modes in various dissipative regimes.

We have shown that the scalings derived for the marginally stable mode, the most unstable wavenumber, and the maximum growth rate with magnetic Reynolds number, $k_{\mathrm{c}}, k_{\max }, \gamma_{\max } \propto \mathrm{Rm}$, valid for resistive, inviscid plasmas, see equations (87), (90) and (91), as well as Sano \& Miyama 1999, also hold when finite Reynolds numbers are involved. This is true as long as the magnetic Prandtl number is of order unity or smaller, as it is usually the case in many astrophysical systems (such as accretion disks around cataclysmic variables and young stellar objects, as well as the Sun) and also in MRI laboratory experiments. Furthermore, we have addressed in detail, for the first time to our knowledge, the physical properties of the MRI in highly viscous, slightly resistive media. These conditions are expected to be found in the hot, diffuse gas in galaxies and galaxy clusters. In this case, we found that the critical wavenumber for the onset of the MRI, the most unstable wavenumber, and the maximum growth rate scale with the Reynolds and magnetic Reynolds numbers according to $k_{\mathrm{c}} \propto(\operatorname{Re} \mathrm{Rm})^{1 / 3}$ and $k_{\max }, \gamma_{\max } \propto \operatorname{Re}^{1 / 2}$, see equations (98), (101) and (102).

We have provided a thorough geometrical description of the viscous, resistive MRI modes in terms of the angles that define the planes containing the velocity and magnetic field perturbations. In the ideal MHD limit, these planes are orthogonal, with the plane containing the velocity disturbances laying at $45^{\circ}$ with respect to the radial direction. We have shown that velocity and magnetic field perturbations are still orthogonal if the magnetic Prandtl number is unity, but that the planes containing them tend to be aligned with the radial and azimuthal directions, respectively, when the Reynolds number increases. In the regime of large Reynolds numbers and small magnetic Reynolds numbers, magnetic and velocity field perturbations tend to be orthogonal and aligned with the azimuthal and radial directions, respectively. On the other hand, in the regime of small Reynolds numbers and large magnetic Reynolds numbers, the perturbed magnetic field tends to be aligned with the azimuthal direction but the velocity field perturbations do not tend to be aligned with the radial direction. The angle between both fields is determined entirely by the epicyclic frequency $\kappa$. It would be very interesting to understand to what extent this geometrical dependence of MRI modes on the Reynolds and magnetic Reynolds numbers influences the physical properties of kinetic and magnetic cells in fully developed viscous, resistive MHD turbulence.

In the ideal MHD limit, the exact (primary) MRI modes are known to be unstable to parasitic (secondary) instabilities (Goodman \& Xu 1994). These parasitic modes have long been suspected to enable the mechanisms that disrupt the primary modes providing an avenue toward saturation. The solutions derived in this work describe the dynamics of primary MRI modes in viscous, resistive media enabling the study of parasitic instabilities for arbitrary combinations of Reynolds and magnetic Reynolds numbers. The modifications in the relative directions of the velocity and magnetic field perturbations characterizing the primary viscous, resistive MRI modes described above can have an important impact on the development and evolution of parasitic instabilities in the presence of dissipation.

We have shown that, for any combination of the Reynolds and magnetic Reynolds numbers, the mean Reynolds stress, $\bar{R}_{r \phi}=\left\langle\delta v_{r}(z, t) \delta v_{\phi}(z, t)\right\rangle$, is always positive and the mean Maxwell stress, $\bar{M}_{r \phi}=\left\langle\delta b_{r}(z, t) \delta b_{\phi}(z, t)\right\rangle$, is always negative. This implies that the mean total stress, $\bar{T}_{r \phi}=\bar{R}_{r \phi}-\bar{M}_{r \phi}$ is always positive, leading always to an outward transport of angular momentum. We have also demonstrated that both the ratio between magnetic and kinetic stresses, $-\bar{M}_{r \phi} / \bar{R}_{r \phi}$, and the ratio between magnetic and kinetic energy densities, $\bar{E}_{M} / \bar{E}_{K}$, are always dominated by the magnetic contribution. These last two statements, support a somewhat unexpected result since it is tempting to think that velocity perturbations would dominate both the transport of angular momentum and 

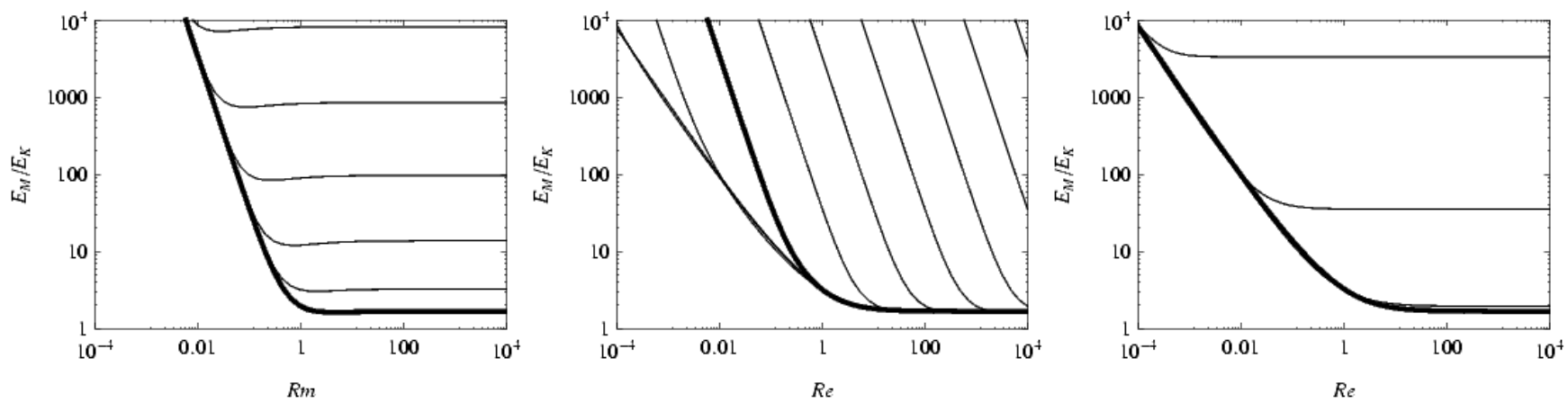

FIG. 11.- Ratio between the magnetic and the kinetic energy densities contained in MRI-driven perturbations, $\bar{E}_{M} / \bar{E}_{K}$, for Keplerian rotation in different dissipative regimes. Left: Ratio between the magnetic and the kinetic energy densities as a function of the magnetic Reynolds number for different values of the Reynolds number. The thick solid line denotes the inviscid limit, i.e., $\operatorname{Re} \rightarrow \infty$. The thin solid lines, in increasing order according to their asymptotic value at high magnetic Reynolds numbers correspond to $R e=1,0.1, \ldots$. For magnetic Reynolds numbers larger than unity, the ratio is independent of Rm regardless of the value of Re. Moreover, the asymptotic value of this ratio for $\mathrm{Rm} \ll 1$ is independent of the Reynolds number. Middle: Ratio between the magnetic and the kinetic energy densities as a function of the Reynolds number for different values of the magnetic Prandtl number. The various curves, from left to right, correspond to $\mathrm{Pm}=10^{2}, 10, \ldots$. The thick solid line corresponds to the case $\mathrm{Pm}=1$. Right: Ratio between the Maxwell and the Reynolds stresses as a function of the Reynolds number for different values of the magnetic Reynolds number. The thick solid line corresponds to the ideal conductor limit, i.e., $\mathrm{Rm} \rightarrow \infty$. The thin solid lines, in increasing order according to their asymptotic value at high Reynolds numbers correspond to $\mathrm{Rm}=1,0.1, \ldots$. Unlike the ratio between stresses, the ratio between energy densities seems to decrease monotonically with Re for any value of the magnetic Reynolds number. The magnetic energy density is larger than the kinetic energy density for any combination of the Reynolds and magnetic Reynolds numbers. The minimum value of this ratio is achieved in the ideal MHD regime, where $E_{M} / \bar{E}_{K}=5 / 3$, for Keplerian rotation.
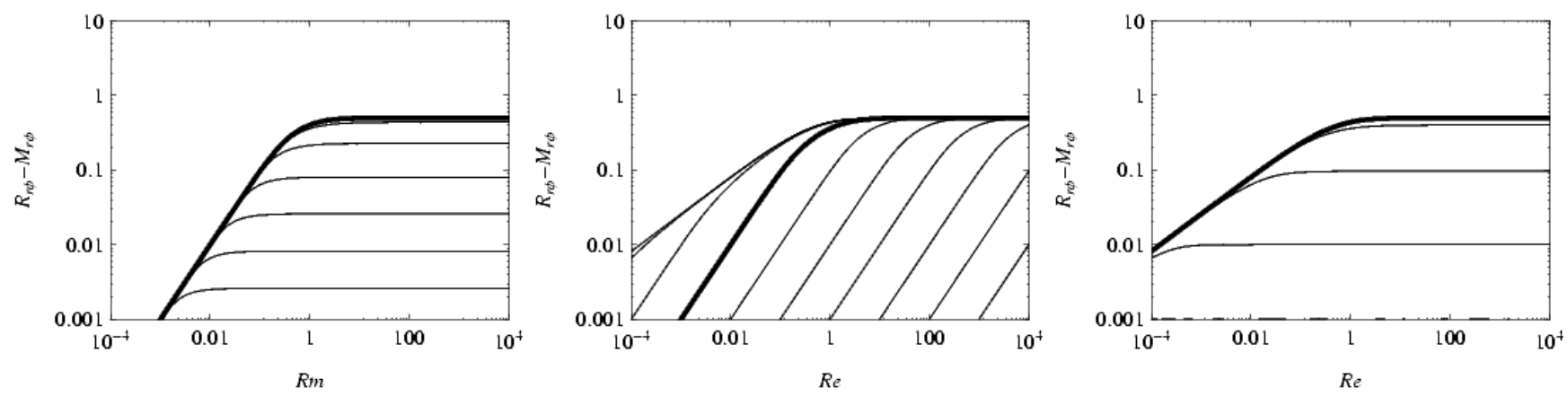

FIG. 12.- Mean total stress responsible for angular momentum transport, $\bar{T}_{r \phi}=\bar{R}_{r \phi}-\bar{M}_{r \phi}$, calculated according to equations 121 and 122 , in various dissipative regimes for Keplerian rotation. Left: Mean total stress $\bar{T}_{r \phi}$ as a function of the magnetic Reynolds number for different values of the Reynolds number. The thick solid line denotes the inviscid limit, i.e., $\operatorname{Re} \rightarrow \infty$. The thin solid lines, in decreasing order, correspond to Re $=10,1, \ldots, 10^{-4}$. For magnetic Reynolds numbers larger than unity, this ratio is independent of $\mathrm{Rm}$ regardless of the value of Re. Moreover, the asymptotic value of this ratio for Rm $\ll 1$ is independent of the Reynolds number. Middle: Mean total stress $\bar{T}_{r \phi}$ as a function of the magnetic Prandtl number for different values of the Reynolds number. From left to right, the curves correspond to $\mathrm{Pm}=10^{3}, 10^{2}, \ldots, 1$ (thick solid line), $\ldots, 10^{-6}$. Right: Mean total stress $\bar{T}_{r \phi}$ as a function of the Reynolds number for different values of the magnetic Reynolds number. The thick solid line corresponds to the ideal conductor limit, i.e., Rm $\rightarrow \infty$. The thin solid lines, in decreasing order, correspond to $\mathrm{Rm}=10,1,0.1$. Note that the stress scale in these plots is arbitrary.

the energy density in highly resistive, inviscid plasmas. It would be very interesting to understand if and how the value of these ratios in the saturated turbulent state vary as a function of the Reynolds and magnetic Reynolds numbers.

Sano and collaborators have studied the linear (Sano \& Mivama 1999) and non-linear (Sano, Inutsuka, \& Miyama 1998; Sano \& Inutsuka 2001; Sano \& Stone 2003; Sano et al. 2004) evolution of the MRI for inviscid, resistive MHD. The simulations in Sano \& Stone (2003) show that for small magnetic Reynolds numbers the stresses at saturation increase rapidly with increasing $\mathrm{Rm}$ and that there exists a critical magnetic Reynolds number, of order unity, beyond which turbulent stresses are rather insensitive to $\mathrm{Rm}$. The fact that this same behavior is indeed seen when the stresses are due to viscous, resistive MRI modes (see Figure 12) rises the question of how strong is the influence of long-lived, channel-like modes on the fully developed turbulent state reached in shearing box simulations with net magnetic flux through the vertical boundaries.
Systematic numerical studies of viscous, resistive MHD shearing flows have begun to uncover the dependencies of microphysical dissipation on the mean transport properties of MRI-driven turbulence. Numerical simulations with both zero (Fromang et al. 2007) and non-zero net magnetic fluxes (Lesur \& Longaretti 2007) lead to the conclusion that angular momentum transport increases with increasing magnetic Prandtl number when the Reynolds number is held constant. This behavior can also be identified when examining the stresses due to viscous, resistive MRI modes, see Figure 12 .

The effects of varying the Reynolds number at fixed magnetic Prandtl number on the saturation of MRI-driven turbulence are currently rather uncertain (see, in particular, the discussion in Lesur \& Longaretti 2007). The global trends exhibited by the available simulations suggest that the stresses at saturation increase with increasing Reynolds number for magnetic Prandtl numbers smaller than unity while the opposite behavior is observed for magnetic Prandtl numbers larger than unity. If confirmed, these results suggest that the mechanisms 
leading to saturation might operate differently depending on whether the magnetic Prandtl number is larger or smaller than unity. In any case, having obtained a better understanding of the behavior of the most unstable MRI modes as a function the magnetic Prandtl number it would be very interesting to follow the evolution of the viscous, resistive MRI from the linear to the non-linear regime. By performing numerical simulations with the same Prandtl number (both larger and smaller than unity) and different Reynolds numbers we could see whether there is an inversion of the trends observed in the linear regime (i.e., higher stresses at higher Reynolds numbers for fixed magnetic Prandtl numbers) after the exact solutions break down. The comparison between the late time behavior of the viscous, resistive MRI modes and fully developed MHD turbulence with dissipation will shed light into the mechanisms that lead to the saturation of the MRI in different dissipative regimes.

Finally, most current numerical algorithms employ finite difference methods (with constrained transport for the evo- lution of the magnetic field). The leading order errors in first-order upwind methods behave like diffusion, however, in second-order central difference methods, the leading order errors are dispersive (artificial viscosities are usually employed to damp unphysical oscillations near shocks). The comparison between numerical solutions from different algorithms can provide estimates of these errors. However, it is difficult to quantify these numerical artifacts based on analytical studies of ideal MHD. The analytical solutions derived in this paper, on the other hand, describe the effects of arbitrary combinations of viscosity and resistivity [see also Lesaffre \& Balbus (2007) who derived results to leading order in $(\eta-\nu) / k]$. It should now be possible to better measure the numerical viscosity and resistivity for a wide range of Reynolds and magnetic Reynolds numbers by comparing numerical solutions and analytical solutions of non-ideal MRI. The results presented in this paper provide ideal benchmarks to the study numerical artifacts generated by different algorithms in various dissipative regimes.

\section{APPENDIX}

\section{ANALYTICAL SOLUTION TO QUARTIC EQUATION AND IDEAL MHD LIMIT}

The solutions to a depressed quartic equation of the form

$$
\sigma^{4}+\alpha \sigma^{2}+\beta \sigma+\lambda=0 .
$$

are given by ${ }^{7}$

$$
\sigma= \pm_{a} \sqrt{-\left(\frac{3 \alpha}{4}+\frac{y}{2} \pm_{b} \frac{\beta / 4}{\sqrt{\alpha / 4+y / 2}}\right)} \pm_{b} \sqrt{\frac{\alpha}{4}+\frac{y}{2}}
$$

where $y$ is any of the solutions of the cubic equation

$$
y^{3}+\frac{5 \alpha}{2} y^{2}+\left(2 \alpha^{2}-\lambda\right) y+\left(\frac{\alpha^{3}}{2}-\frac{\alpha \lambda}{2}-\frac{\beta^{2}}{8}\right)=0 .
$$

In the special case $\beta \rightarrow 0$, the solutions to equation (A3) take simple forms. In order to see how the general solutions reduce to the simple cases, we write the cubic equation as

$$
\left(y+\frac{\alpha}{2}\right)\left[(y+\alpha)^{2}-\lambda\right]=\frac{\beta^{2}}{8} .
$$

It is easy to see that, if $y \neq-\alpha / 2$,

$$
\frac{\beta / 4}{\sqrt{\alpha / 4+y / 2}}=\sqrt{(y+\alpha)^{2}-\lambda}
$$

Using the above identity, the general solution (A2) becomes

$$
\sigma= \pm_{a} \sqrt{-\Lambda \pm_{b} \sqrt{\Delta}} \pm_{b} \frac{\beta / 4}{\sqrt{\Delta}}
$$

where we have defined

$$
\Lambda=\frac{3 \alpha}{4}+\frac{y}{2} \quad \text { and } \quad \Delta=\sqrt{(y+\alpha)^{2}-\lambda}
$$

If we choose the root so that

$$
\lim _{\beta \rightarrow 0} y=-\frac{\alpha}{2}
$$

it is easier to take the limit

$$
\lim _{\beta \rightarrow 0} \sigma=\lim _{\beta \rightarrow 0} \pm \sqrt{-\Lambda \pm \sqrt{\Delta}}= \pm \sqrt{-\frac{\alpha}{2} \mp \sqrt{\frac{\alpha^{2}}{4}-\lambda}} \equiv \pm \sqrt{-\Lambda_{0} \pm \sqrt{\Delta_{0}}}=\sigma_{0}
$$

\footnotetext{
${ }^{7}$ Note that the two $\pm_{b}$ 's have the same sign so there are only four solutions instead of eight.
} 
where $\Lambda_{0}$ and $\Delta_{0}$ are defined in equations (74) and (75).

We thank Jeremy Goodman and Dimitrios Psaltis for valuable comments and discussions. We are grateful to Roman Shcherbakov for the initial discussions that lead to the idea of combining the dispersion relation and its derivative to obtain the asymptotic expressions derived in $\S 4$ MEP gratefully acknowledges support from the Institute for Advanced Study. CKC is supported through an ITC Fellowship at Harvard. MEP and CKC are grateful to the Harvard-Smithsonian Institute for Theory and Computation and the Institute for Advanced Study, respectively, for their hospitality during part of this work.

\section{REFERENCES}

Balbus, S. A. \& Hawley J. F. 1991, ApJ, 376, 214

Balbus, S. A. \& Hawley J. F. 1992, ApJ, 392, 662 1998, Rev. Mod. Phys., 70, 1

Balbus, S. A. \& Henri, P. 2008, ApJ, in press [arXiv0706.0828]

Blaes, O. M., \& Balbus, S. A. 1994, ApJ, 421, 163

Brandenburg, A., Nordlund, A., Stein, R. F., \& Torkelsson, U. 1995, ApJ, 446, 741

Fleming, T. P., Stone, J. M., Hawley 2000, ApJ, 530, 464

Fromang, S. \& Papaloizou, J. 2007, A\&A 476, 1113

Fromang, S., Papaloizou, J., Lesur, G., \& Heinemann, T. 2007, A\&A, 476, 1123

Gammie, C. F. 1996, ApJ, 462, 725

Goodman, J. \& Xu, G. 1994, ApJ, 432, 213

Goodman, J. \& Ji, H. 2002, JFM, 462, 365

Hawley, J. F., Gammie, C. F., \& Balbus, S. A. 1995, ApJ, 440, 742

Hoffman K.M., Kunze R., 1971, Linear Algebra, 2nd ed. Prentice Hall, N.J.

Ji, H., Goodman, J. \& Kageyama, A. 2001, MNRAS, 325, L1

Jin, L. 1996, ApJ, 457, 798

Liu, W., Goodman, J. \& Ji, H. 2006, ApJ, 643, 306

Lesur, G. \& Longaretti, P. Y. 2007, MNRAS, 378, 1471
Lesaffre, P. \& Balbus, S. A. 2007, MNRAS, 381, 319

Pessah, M. E. \& Psaltis, D. 2005, ApJ, 628, 879

Pessah, M. E., Chan C. K., \& Psaltis, D. 2006, MNRAS, 372, 183

Pessah, M. E., Chan C. K., \& Psaltis, D. 2007, ApJ, 668, L51

Rüdiger, G., Schultz, M., \& Shalybkov, D. 2003, Phys. Rev. E, 67, 046312

Salmeron, R. \& Wardle, M. 2005, MNRAS, 361, 45

Sano, T. \& Inutsuka, S. I. 2001, ApJ, 561, L179

Sano, T., Inutsuka, S. I., \& Miyama, S. M. 1998, ApJ, 506, L57

Sano, T., Inutsuka, S. I., Turner, N. J., \& Stone, J. M. 2004, ApJ, 605, 321

Sano, T. \& Miyama, S. M. 1999, ApJ, 515, 776

Sano, T. \& Stone, J. M. 2003, in Scientific Frontiers in Research on Extrasolar Planets, ASPC, Vol 294, D., Deming \& S., Seager, eds. (San Francisco: ASP)

Sisan, D. R. Mujica, N., Tillotson, W. A., Huang, Y. M., Dorland, W., Hassam, A. B., Antonsen, T. M., \& Lathrop, D. P. 2004, Phys. Rev. Lett., 93, 114502

Turner, N. J., Sano, T. \& Dziourkevitch, N. 2007, ApJ, 659, 729 